\title{
GENERALIZING CIRCLES OVER ALGEBRAIC EXTENSIONS
}

\author{
T. RECIO, J. R. SENDRA, L. F. TABERA, AND C. VILLARINO
}

\begin{abstract}
This paper deals with a family of spatial rational curves that were introduced in 1999 by Andradas, Recio, and Sendra, under the name of hypercircles, as an algorithmic cornerstone tool in the context of improving the rational parametrization (simplifying the coefficients of the rational functions, when possible) of algebraic varieties. A real circle can be defined as the image of the real axis under a Moebius transformation in the complex field. Likewise, and roughly speaking, a hypercircle can be defined as the image of a line ("the $\mathbb{K}$-axis") in an $n$-degree finite algebraic extension $\mathbb{K}(\alpha) \approx \mathbb{K}^{n}$ under the transformation $\frac{a t+b}{c t+d}: \mathbb{K}(\alpha) \rightarrow \mathbb{K}(\alpha)$.

The aim of this article is to extend, to the case of hypercircles, some of the specific properties of circles. We show that hypercircles are precisely, via $\mathbb{K}$-projective transformations, the rational normal curve of a suitable degree. We also obtain a complete description of the points at infinity of these curves (generalizing the cyclic structure at infinity of circles). We characterize hypercircles as those curves of degree equal to the dimension of the ambient affine space and with infinitely many $\mathbb{K}$-rational points, passing through these points at infinity. Moreover, we give explicit formulae for the parametrization and implicitation of hypercircles. Besides the intrinsic interest of this very special family of curves, the understanding of its properties has a direct application to the simplification of parametrizations problem, as shown in the last section.
\end{abstract}

\section{INTRODUCTION}

The problem of obtaining a real parametrization of a rational planar curve given by a complex parametrization has been studied, from an algorithmic point of view, in 10. There, the problem is reduced to determining that a certain curve obtained after manipulating the given parametrization is a real line or a real circle. From a real parametrization of this circle (or line), a real parametrization of the original curve is then achieved. This auxiliary circle is found by an analogous to Weil descente's method [19] applied to the complex parametrization of the curve originally given. In [4], the same approach has been extended to the general case of planar or spatial rational curves $\mathcal{C}$ given by a parametrization over $\mathbb{K}(\alpha)$, where $\alpha$ is an algebraic element over $\mathbb{K}$. In order to obtain, whenever possible, a parametrization over $\mathbb{K}$ of $\mathcal{C}$, another rational curve, with remarkable properties, is associated to $\mathcal{C}$.

Received by the editor November 2, 2006 and, in revised form, August 2, 2008.

2000 Mathematics Subject Classification. Primary 14Q05, Secondary 14M20.

The authors are partially supported by the project MTM2005-08690-C02-01/02.

The second and fourth authors were partially supported by CAM-UAH2005/053 "Dirección General de Universidades de la Consejería de Educación de la CAM y la Universidad de Alcalá".

The third author was also supported by a FPU research grant. Ministerio de Educación y Ciencia. 
In 4 it is shown that this associated curve is, in the relevant cases, a generalization of a circle, in the sense we will discuss below, deserving to be named hypercircle.

The simplest hypercircles should be the circles themselves. We can think of the real plane as the field of complex numbers $\mathbb{C}$, an algebraic extension of the reals $\mathbb{R}$ of degree 2. Analogously, we can consider a characteristic zero base field $\mathbb{K}$ and an algebraic extension of degree $n, \mathbb{K}(\alpha)$. Let us identify $\mathbb{K}(\alpha)$ as the vector space $\mathbb{K}^{n}$, via the choice of a suitable base, such as the one given by the powers of $\alpha$. This is the framework in which hypercircles are defined.

Now let us look at the different, equivalent, ways of defining a common circle on the real plane, with the purpose of taking the most convenient one for generalization. The first definition of a circle is the set of points in the real plane that are equidistant from a fixed point. This approach does not extend well to more general algebraic extensions, because we do not have an immediate notion of metric over $\mathbb{K}^{n}$. On the other hand, algebraically, a real planar circle is a conic such that its homogeneous degree two form is $x^{2}+y^{2}$ and such that it contains an infinite number of real points. Even if we will prove in Section 6 that we can show an analogous definition for a hypercircle, this is not an operative way to start defining them.

Finally, from another point of view, we see that circles are real rational curves. This means that there are two real rational functions $\left(\phi_{1}(t), \phi_{2}(t)\right)$ whose images cover almost all the points of the circle. For instance, the circle $x^{2}+y^{2}=1$ is parametrized by $\phi(t)=\left(\frac{t^{2}-1}{t^{2}+1}, \frac{2 t}{t^{2}+1}\right)$. Every proper (almost one-to-one [15]) rational parametrization of a circle verifies that $\phi_{1}(t)+i \phi_{2}(t)=\frac{a t+b}{c t+d} \in \mathbb{C}(t) \backslash \mathbb{C}$, which defines a conformal mapping $u: \mathbb{C} \rightarrow \mathbb{C}$. Moreover, if we identify $\mathbb{C}$ with $\mathbb{R}^{2}$, the image of the real axis $(t, 0)$ under $u$ is exactly the circle parametrized by $\phi(t)$. Conversely, let $u(t)=\frac{a t+b}{c t+d} \in \mathbb{C}(t)$ be a unit of the near-ring $\mathbb{C}(t)$ under the composition operator (see [20]). If $c \neq 0$ and $d / c \notin \mathbb{R}$, then the closure of the image by $u$ of the real axis is a circle; otherwise, it is a line. This method to construct circles generalizes easily to algebraic extensions. Namely, let $u(t)=\frac{a t+b}{c t+d}$ be a unit of $\mathbb{K}(\alpha)(t)$ (i.e. verifying that $a d-b c \neq 0$ ). Let us identify $\mathbb{K}(\alpha)$ with $\mathbb{K}^{n}$ and let $u$ be the map

$$
\begin{array}{ccc}
u: \quad \mathbb{K}(\alpha) \approx \mathbb{K}^{n} & \rightarrow & \mathbb{K}(\alpha) \approx \mathbb{K}^{n} \\
t & \mapsto & u(t) .
\end{array}
$$

Then, the Zariski-closure of the image of the axis $(t, 0, \ldots, 0)$ under the map $u$ is a rational curve in $\mathbb{K}^{n}$. These curves are, by definition, our hypercircles.

Roughly speaking, it happens (see 4]) that a parametrization over $\mathbb{K}$ of the hypercircle associated to a given rational curve $\mathcal{C}$ (whose parametrization we want to simplify) can be used to get, in a straightforward manner, a parametrization of $\mathcal{C}$ over $\mathbb{K}$. As pointed out in 4 , it seems that, due to the geometric properties of hypercircles, it is algorithmically simpler to obtain such a parametrization for this type of curve than it is for $\mathcal{C}$. In fact, it is shown in [12] how to get this in some cases. Therefore, the reparametrization problem is behind our increasing interest in the study of hypercircles on its own.

The structure of this paper is as follows. In Section 2 we formally introduce the notion of hypercircle. We study the influence on a hypercircle when adding and multiplying the defining unit $u(t)$ by elements of $\mathbb{K}(\alpha)$, reducing the affine classification of hypercircles to those defined by some simpler units. Next we characterize the units associated to lines. In Section 3 we show how to transform, projectively, a hypercircle into the rational normal curve (see [6]). From this, we derive the 
main geometric properties of hypercircles (smoothness, degree, affine equivalence, etc.) and we reduce the study of hypercircles to the subclass of primitive hypercircles (See Definition 3.5). In Section 4 the behavior of hypercircles at infinity is analyzed, showing its precise and rich structure. In Section 5, exploiting the stated geometric features, we present ad hoc parametrization and implicitization methods for hypercircles. In Section 6 we characterize hypercircles among curves of degree equal to the dimension of the ambient affine space, passing through the prescribed points of infinity described in Section 4 and having infinitely many rational points. Finally, Section 7 is devoted to showing how the insight gained throughout this paper can be applied to derive heuristics for solving the problem of simplifying the parametrization of curves with coefficients involving algebraic elements.

Throughout this paper the following notation and terminology will be used.

- $\mathbb{K}$ will be a field of characteristic zero, $\mathbb{K} \subseteq \mathbb{L}$ a finite algebraic extension of degree $n$ and $\mathbb{F}$ the algebraic closure of $\mathbb{K}$.

- $\alpha$ will be a primitive element of $\mathbb{L}$ over $\mathbb{K}$.

- $u(t)$ will be a unit under composition of $\mathbb{L}(t)$. That is, $u(t)=\frac{a t+b}{c t+d}$ with $a d-b c \neq 0$. Its inverse $\frac{-d t+b}{c t-a}$ is denoted by $v(t)$.

- For $u(t)=\frac{a t+b}{c t+d}$ and $c \neq 0, M(t)=t^{r}+k_{r-1} t^{r-1}+\cdots+k_{0} \in \mathbb{K}[t]$ denotes the minimal polynomial of $-d / c$ over $\mathbb{K}$.

- We will denote as $m(t)$ the polynomial obtained by dividing $M(t)$ by $c t+d$. That is, $m(t)=\frac{M(t)}{c t+d}=l_{r-1} t^{r-1}+l_{r-2} t^{r-2}+\cdots+l_{0} \in \mathbb{L}[t]$.

- Sometimes we will represent $u(t)$ as

$$
u(t)=\frac{(a t+b) m(t)}{M(t)}=\frac{p_{0}(t)+p_{1}(t) \alpha+\cdots+p_{n-1}(t) \alpha^{n-1}}{M(t)},
$$

where $p_{i}(t) \in \mathbb{K}[t]$.

- By $\left\{\sigma_{1}=I d, \sigma_{2}, \ldots, \sigma_{s}\right\}, s \geq n$ we will denote the group of $\mathbb{K}$ automorphisms of the normal closure of $\mathbb{K} \subseteq \mathbb{L}$.

- We will represent by $\left\{\alpha_{1}=\alpha, \ldots, \alpha_{n}\right\}$ the conjugates of $\alpha$. We assume, without loss of generality, that $\sigma_{i}(\alpha)=\alpha_{i}$ for $i=1, \ldots, n$.

\section{Definition AND FIRST PROPERTIES}

In this section we begin with the formal definition of a hypercircle.

Definition 2.1. Let $u(t)$ be a unit in $\mathbb{L}(t)$, where $\mathbb{L}=\mathbb{K}(\alpha)$. Let

$$
u(t)=\sum_{i=0}^{n-1} \phi_{i}(t) \alpha^{i}
$$

where $\phi_{i}(t) \in \mathbb{K}(t)$, for $i=0, \ldots, n-1$. The $\alpha$-hypercircle $\mathcal{U}$ generated by $u(t)$ is the rational curve in $\mathbb{F}^{n}$ parametrized by $\phi(t)=\left(\phi_{0}(t), \ldots, \phi_{n-1}(t)\right)$.

Observe that the expansion of $u(t)$ in powers of $\alpha$ is unique, because $\{1, \alpha$, $\left.\ldots, \alpha^{n-1}\right\}$ is a basis of $\mathbb{K}(\alpha)(t)$ as a $\mathbb{K}(t)$ vector space. The parametrization can be obtained by rationalizing the denominator as follows: suppose given the unit $u(t)=\frac{a t+b}{c t+d}, c \neq 0$ (note that, if $c=0$, it is straightforward to obtain $\phi(t)$ ), and the extension $\mathbb{K} \subseteq \mathbb{K}(\alpha)$. Let $M(t)$ be the minimal polynomial of $-d / c$ over $\mathbb{K}$. 


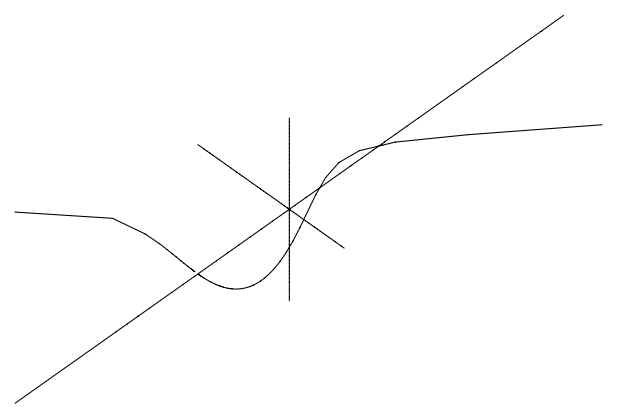

Figure 1. A hypercircle in $\mathbb{R}^{3}$

Compute the quotient $m(t)=\frac{M(t)}{c t+d} \in \mathbb{K}(\alpha)[t]$ and develop the unit as

$$
\frac{a t+b}{c t+d}=\frac{(a t+b) m(t)}{M(t)}=\frac{p_{0}(t)+p_{1}(t) \alpha+\cdots+p_{n-1}(t) \alpha^{n-1}}{M(t)}
$$

where $p_{i}(t) \in \mathbb{K}[t]$. From this, $\phi(t)=\left(\frac{p_{0}(t)}{M(t)}, \ldots, \frac{p_{n-1}(t)}{M(t)}\right)$ is the parametrization associated to $u(t)$. Note that $\operatorname{gcd}\left(p_{0}(t), \ldots, p_{n-1}(t), M(t)\right)=1$. Moreover, it is clear that $\mathbb{F}\left(\phi_{0}(t), \ldots, \phi_{n-1}(t)\right)=\mathbb{F}(t)$. So this parametrization is proper in $\mathbb{F}$, and it follows from the results in [1] that $\mathbb{K}\left(\phi_{0}(t), \ldots, \phi_{n-1}(t)\right)=\mathbb{K}(t)$ also.

Example 2.2. Let us consider the algebraic extension $\mathbb{Q} \subseteq \mathbb{Q}(\alpha)$, where $\alpha^{3}+2 \alpha+$ $2=0$. The unit $\frac{t-\alpha}{t+\alpha}$ has an associated hypercircle parametrized by

$$
\phi(t)=\left(\frac{t^{3}+2 t+2}{t^{3}+2 t-2}, \frac{-2 t^{2}}{t^{3}+2 t-2}, \frac{2 t}{t^{3}+2 t-2}\right)
$$

A picture of the spatial real curve is shown in Figure 1

As it stands, the definition of a hypercircle $\mathcal{U}$ depends on a given unit $u(t) \in \mathbb{L}(t)$ and on a primitive generator $\alpha$ of an algebraic extension $\mathbb{L}$. In what follows we will analyze the effect on $\mathcal{U}$ when varying some of these items, searching for a simple representation of a hypercircle to make it easier to study its geometry.

First notice that, given a unit $u(t) \in \mathbb{L}(t)$ and two different primitive elements $\alpha$ and $\beta$ of the extension $\mathbb{K} \subseteq \mathbb{L}$, we can expand the unit in two different ways as $u(t)=\sum_{i=0}^{n-1} \alpha^{i} \phi_{i}(t)=\sum_{i=0}^{n-1} \beta^{i} \psi_{i}(t)$. The hypercircles $\mathcal{U}_{\alpha} \simeq\left(\phi_{0}(t), \ldots, \phi_{n-1}(t)\right)$ and $\mathcal{U}_{\beta} \simeq\left(\psi_{0}(t), \ldots, \psi_{n-1}(t)\right)$ generated by $u(t)$ are different curves in $\mathbb{F}^{n}$; see Example 2.3. Nevertheless, let $\mathcal{A} \in \mathcal{M}_{n \times n}(\mathbb{K})$ be the matrix of a change of basis from $\left\{1, \alpha, \ldots, \alpha^{n-1}\right\}$ to $\left\{1, \beta, \ldots, \beta^{n-1}\right\}$. Then, $\mathcal{A}\left(\phi_{0}(t), \ldots, \phi_{n-1}(t)\right)^{t}=$ $\left(\psi_{0}(t), \ldots, \psi_{n-1}(t)\right)^{t}$. That is, it carries one curve onto the other. Thus, $\mathcal{U}_{\alpha}$ and $\mathcal{U}_{\beta}$ are related by the affine transformation induced by the change of basis and, so, they share many important geometric properties.

In the sequel, if there is no confusion about the algebraic extension and the primitive element, we will simply call $\mathcal{U}$ a hypercircle.

Example 2.3. Let us consider the algebraic extension $\mathbb{Q} \subseteq \mathbb{Q}(\alpha)$, where $\alpha^{4}+1=0$. Let us take the unit $u(t)=\frac{t-\alpha}{t+\alpha}$. By normalizing $u(t)$, we obtain the parametrization $\phi(t)$ associated to $u(t)$ :

$$
\phi(t)=\left(\frac{t^{4}-1}{t^{4}+1}, \frac{-2 t^{3}}{t^{4}+1}, \frac{2 t^{2}}{t^{4}+1}, \frac{-2 t}{t^{4}+1}\right) .
$$


This hypercircle $\mathcal{U}_{\alpha}$ is the zero set of $\left\{X_{1} X_{2}-X_{3} X_{0}-X_{3}, X_{1}^{2}+X_{3}^{2}-2 X_{2}, X_{1} X_{0}+\right.$ $\left.X_{2} X_{3}-X_{1}, X_{0}^{2}+X_{3} X_{1}-1\right\}$. Now, we take $\beta=\alpha^{3}+1$, instead of $\alpha$, as the primitive element of $\mathbb{Q}(\alpha)=\mathbb{Q}(\beta)$. The same unit $u(t)$ generates the $\beta$-hypercircle $\mathcal{U}_{\beta}$ parametrized by

$$
\psi(t)=\left(\frac{t^{4}+2 t^{3}-2 t^{2}+2 t-1}{t^{4}+1}, \frac{-6 t^{3}+4 t^{2}-2 t}{t^{4}+1}, \frac{6 t^{3}-2 t^{2}}{t^{4}+1}, \frac{-2 t^{3}}{t^{4}+1}\right),
$$

which is different than $\mathcal{U}_{\alpha}$; note that $\psi(1)=(1,-2,2,-1)$ does not satisfy the equation $X_{0}^{2}+X_{3} X_{1}-1=0$ of $\mathcal{U}_{\alpha}$.

On the other hand, it is well known that a given parametric curve can be parametrized over a given field $\mathbb{S}$ by different proper parametrizations, precisely, those obtained by composing to the right a given proper parametrization by a unit in $\mathbb{S}(t)$. In this way, we have a bijection between $\alpha$-hypercircles and the equivalence classes of units of $\mathbb{K}(\alpha)(t)$ under the equivalence relation " $u \sim v$ iff $u(t)=v(\tau(t))$ for a unit $\tau(t) \in \mathbb{K}(t)$ " (fixing the correspondence, between a unit in $\mathbb{K}(\alpha)(t)$ and a hypercircle, by means of the expansion of the unit in terms of powers of $\alpha$ ).

More interesting is to analyze, on a hypercircle defined by a unit $u(t)$, the effect of composing it to the left with another unit $\tau(t) \in \mathbb{K}(\alpha)(t)$, that is, of getting $\tau(u(t))$. For instance, $\tau(t)$ could be $\tau(t)=t+\lambda$ or $\tau(t)=\lambda t$, or $\tau(t)=1 / t$, with $\lambda \in \mathbb{K}(\alpha)^{*}$. Every unit is a sequence of compositions of these three simpler cases; for instance, when $c \neq 0$, we have

$$
\begin{gathered}
t \longmapsto c t \longmapsto c t+d \longmapsto \frac{1}{c t+d} \longmapsto \frac{b c-a d}{c} \frac{1}{c t+d} \\
\longmapsto \frac{a}{c}+\frac{b c-a d}{c} \frac{1}{c t+d}=\frac{a t+b}{c t+d}=u(t) .
\end{gathered}
$$

Therefore, studying their independent effect is all we need to understand completely the behavior of a hypercircle under left composition by units.

For circles, adding a complex number to the unit that defines the circle corresponds to a translation of the circle. Multiplying it by a complex number acts as the composition of a rotation and a dilation. And the application $\tau(t)=1 / t$ gives an inversion. The following lemma analyzes what happens in the general case.

Lemma 2.4. Let $\mathcal{U}$ be the $\alpha$-hypercircle generated by $u(t)$, and $\lambda=\sum_{i=0}^{n-1} \lambda_{i} \alpha^{i} \in$ $\mathbb{K}(\alpha)^{*}$, where $\lambda_{i} \in \mathbb{K}$. Then,

(1) $\lambda+u(t)$ is a unit generating the hypercircle obtained from $\mathcal{U}$ by the translation of vector $\left(\lambda_{0}, \ldots, \lambda_{n-1}\right)$.

(2) $\lambda u(t)$ is a unit generating the hypercircle obtained from $\mathcal{U}$ by the affine transformation over $\mathbb{K}$ given by the matrix of a change of basis from $\mathcal{B}^{\star}=$ $\left\{\lambda, \lambda \alpha, \ldots, \lambda \alpha^{n-1}\right\}$ to $\mathcal{B}=\left\{1, \alpha, \ldots, \alpha^{n-1}\right\}$.

Proof. To prove (1), let $\phi(t)=\left(\phi_{0}(t), \ldots, \phi_{n-1}(t)\right) \in \mathbb{K}(t)^{n}$ be the parametrization of $\mathcal{U}$ obtained from $u(t)$. Then, $\lambda+u(t)=\sum_{i=0}^{n-1}\left(\lambda_{i}+\phi_{i}(t)\right) \alpha^{i}$ generates the hypercircle parametrized by $\left(\lambda_{0}+\phi_{0}(t), \ldots, \lambda_{n-1}+\phi_{n-1}(t)\right) \in \mathbb{K}(t)^{n}$, which is the translation of $\mathcal{U}$ of vector $\left(\lambda_{0}, \ldots, \lambda_{n-1}\right)$. For the second assertion, let $\phi^{\star}(t) \in \mathbb{K}(t)^{n}$ be the parametrization of the hypercircle associated to the unit $\lambda u(t)$. The rational coordinates $\phi_{i}^{\star}(t)$ of $\phi^{\star}(t)$ are obtained from the matrix $\mathcal{A}=\left(a_{i, j}\right) \in \mathcal{M}_{n \times n}(\mathbb{K})$ of 
a change of basis from $\mathcal{B}^{\star}$ to $\mathcal{B}$, for $i, j=0, \ldots, n-1$. Indeed,

$$
\lambda u(t)=\sum_{i=0}^{n-1} \phi_{i}(t) \lambda \alpha^{i}=\sum_{i=0}^{n-1} \phi_{i}(t)\left(\sum_{j=0}^{n-1} a_{j i} \alpha^{j}\right)=\sum_{j=0}^{n-1}\left(\sum_{i=0}^{n-1} a_{j i} \phi_{i}(t)\right) \alpha^{j} .
$$

Then $\phi^{\star}(t)^{t}=\mathcal{A} \phi(t)^{t}$.

Finally, the following lemma uses the previous results to transform affinely one hypercircle into another one whose unit is simpler.

Lemma 2.5. Let $u(t)=\frac{a t+b}{c t+d}$ be a unit and $\mathcal{U}$ its associated hypercircle.

(1) If $c=0$, then $\mathcal{U}$ is affinely equivalent over $\mathbb{K}$ to the line generated by $u^{\star}(t)=t$.

(2) If $c \neq 0$, then $\mathcal{U}$ is affinely equivalent over $\mathbb{K}$ to the hypercircle $\mathcal{U}^{\star}$ generated by $u^{\star}(t)=\frac{1}{t+d / c}$.

Proof. This lemma follows from Lemma 2.4 taking into account that $u(t)$ is obtained from $u^{\star}(t)$ by the composition

$$
u^{\star}(t) \mapsto \lambda_{1} u^{\star}(t) \mapsto \lambda_{1} u^{\star}(t)+\lambda_{2}=u(t)
$$

with suitable $\lambda_{1}, \lambda_{2}, u^{\star}$. If $c=0$, then $\lambda_{1}=\frac{a}{d} \neq 0$ and $\lambda_{2}=\frac{b}{d}$ for $u^{\star}(t)=t$. Analogously, if $c \neq 0$, then $u(t)$ is obtained from $u^{\star}(t)=\frac{1}{t+d / c}$ taking $\lambda_{1}=\frac{b c-a d}{c^{2}} \neq$ 0 and $\lambda_{2}=\frac{a}{c}$.

Therefore the (affine) geometry of hypercircles can be reduced to those generated by a unit of type $\frac{1}{t+d}$ (then we say the unit is in reduced form). The simplest hypercircle of this kind is given by $\frac{1}{t+d}$, when $d \in \mathbb{K}$. It is the line parametrized by $\left(\frac{1}{t+d}, 0, \ldots, 0\right)$. In the complex case, Moebius transformations defining lines are precisely those given either by a polynomial unit in $t$ (i.e. a unit without $t$ at the denominator) or by a unit such that the root of the denominator is in $\mathbb{R}$. The same property holds for hypercircles.

Theorem 2.6. Let $\mathcal{U}$ be the $\alpha$-hypercircle associated to $u(t)$. Then, the following statements are equivalent:

(1) $\mathcal{U}$ is a line.

(2) $\mathcal{U}$ is associated to a polynomial unit.

(3) The root of the denominator of every nonpolynomial unit generating $\mathcal{U}$ belongs to $\mathbb{K}$.

(4) $\mathcal{U}$ is polynomially parametrizable (over $\mathbb{F}$ ).

(5) $\mathcal{U}$ has one and only one branch (over $\mathbb{F}$ ) at infinity.

(6) $\mathcal{U}$ is polynomially parametrizable over $\mathbb{K}$.

(7) $\mathcal{U}$ has one and only one branch (over $\mathbb{K}$ ) at infinity.

Proof. (1) $\Leftrightarrow(2)$. By definition, we know that hypercircles have a parametrization over $\mathbb{K}$. Thus, if $\mathcal{U}$ is a line, it can be parametrized as $\left(a_{0} t+b_{0}, \ldots, a_{n-1} t+\right.$ $\left.b_{n-1}\right)$, where $a_{i}, b_{i} \in \mathbb{K}$. Therefore, $u(t)=\left(\sum_{i=0}^{n-1} a_{i} \alpha^{i}\right) t+\sum_{i=0}^{n-1} b_{i} \alpha^{i}$ is a polynomial unit associated to $\mathcal{U}$. Conversely, let $u(t)=a t+b \in \mathbb{L}(t), a \neq 0$, be a polynomial unit associated to $\mathcal{U}$. Then $\mathcal{U}$ is the line parametrized by $\mathcal{P}(t)=$ $\left(a_{0} t+b_{0}, \ldots, a_{n-1} t+b_{n-1}\right) \in \mathbb{K}[t]^{n}$, where $a=\sum_{i=0}^{n-1} a_{i} \alpha^{i}$ and $b=\sum_{i=0}^{n-1} b_{i} \alpha^{i}$.

$(2) \Leftrightarrow(3)$. Let $u(t)=a t+b$ be a polynomial unit associated to $\mathcal{U}$, and let $u^{\star}(t)$ be another nonpolynomial unit associated to $\mathcal{U}$. Then, $u^{\star}(t)=u(\tau(t))$, where $\tau(t)$ 
is a unit of $\mathbb{K}(t)$. Therefore, the root of $u^{\star}(t)$ belongs to $\mathbb{K}$. Conversely, by Lemma 2.5. (3) implies (1), and we know that (1) implies (2).

$(3) \Leftrightarrow(4)$. Indeed, (3) implies (2) and therefore (4). Conversely, let $u(t)$ be a nonpolynomial unit generating $\mathcal{U}$, and let $\phi(t)=\left(\phi_{i}\right)_{i=1, \ldots, n} \in \mathbb{K}(t)^{n}$ be the associated parametrization of $\mathcal{U}$. Then, $\phi(t)$ is proper, $\phi_{i}(t)=\frac{p_{i}(t)}{M(t)}$ with $\operatorname{deg}\left(p_{i}\right) \leq \operatorname{deg}(M)$ and $\operatorname{gcd}\left(p_{0}(t) \ldots p_{n-1}(t), M(t)\right)=1$. Thus, the fact that $\mathcal{U}$ admits a polynomial parametrization, implies, by Abhyankar-Manocha-Canny's criterion of polynomiality (see [9]), that the denominator $M(t)$ is either constant or has only one root. Now, $M(t)$ cannot be constant, since it is a minimal polynomial. Thus, $M$ has only one root, and since it is irreducible, it must be linear. Moreover, since $M \in \mathbb{K}[t]$, its root is an element in $\mathbb{K}$.

$(4) \Leftrightarrow(5)$. This is, again, the geometric version of Abhyankar-Manocha-Canny's criterion. The same for $(6) \Leftrightarrow(7)$.

$(4) \Leftrightarrow(6)$. Obviously (6) implies (4). Conversely, if we have a polynomial parametrization over $\mathbb{F}$, it happens 2 that any proper parametrization must be either polynomial or in all its components the degree of the numerator must be smaller or equal than the degree of the denominator and, then, this denominator has only one single root over $\mathbb{F}$. So, since the parametrization $\phi(t)$ induced by the unit is proper, and by hypothesis $\mathcal{U}$ is polynomial, then $\phi(t)$ must be either polynomial (in which case we are done because $\phi(t)$ is over $\mathbb{K}$ ) or its denominator $M(t)$ has a single root $a \in \mathbb{F}$. Now, by reasoning as above one gets that $a \in \mathbb{K}$. So, a change of parameter, such as $t \mapsto \frac{1+a s}{s}$ turns $\phi(t)$ into a $\mathbb{K}$-polynomial parametrization.

As a corollary of this theorem, we observe that a parabola can never be a hypercircle, since it is polynomially parametrizable, but it is not a line. Nevertheless, it is easy to check that the other irreducible conics are indeed hypercircles for certain algebraic extensions of degree 2 .

\section{Main geOMetric PROPERTIES}

This section is devoted to the analysis of the main geometric properties of hypercircles. The key idea, when not dealing with lines, will be to use the reduction to units of the form $u(t)=\frac{1}{t+d}$, where $d \notin \mathbb{K}$ (see Lemma 2.5).

Theorem 3.1. Let $\mathcal{U}$ be the $\alpha$-hypercircle associated to the unit $u(t)=\frac{a t+b}{t+d} \in$ $\mathbb{K}(\alpha)(t)$ and let $r=[\mathbb{K}(-d): \mathbb{K}]$. Then:

(1) There exists an affine transformation $\chi: \mathbb{F}^{n} \longrightarrow \mathbb{F}^{n}$ defined over $\mathbb{K}$ such that the curve $\chi(\mathcal{U})$ is parametrized by

$$
\tilde{\chi}(t)=\left(\frac{1}{M(t)}, \frac{t}{M(t)}, \ldots, \frac{t^{r-1}}{M(t)}, 0, \ldots, 0\right) .
$$

(2) There exists a projective transformation $\rho: \mathbb{P}(\mathbb{F})^{n} \longrightarrow \mathbb{P}(\mathbb{F})^{n}$, defined over $\mathbb{K}$ such that the curve $\rho(\mathcal{U})$ is the rational normal curve of degree $r$ in $\mathbb{P}(\mathbb{F})^{n}$ parametrized by

$$
\widetilde{\rho}(t: s)=\left[s^{r}: s^{r-1} t: \cdots: s t^{r-1}: t^{r}: 0: \cdots: 0\right] .
$$

Proof. For the case of lines the result is trivial. By Lemma 2.5, we can consider that $\mathcal{U}$ is the hypercircle associated to $u(t)=\frac{1}{t+d}$ and $r \geq 2$. Let $M(t)=t^{r}+k_{r-1} t^{k-1}+$ $\cdots+k_{0} \in \mathbb{K}[t], m(t)=\sum_{i=0}^{r-1} l_{i} t^{i} \in \mathbb{L}[t]$, as indicated in Section 1 and, since the numerator of $u(t)$ is 1 , it holds that $m(t)=\sum_{i=0}^{n-1} p_{i}(t) \alpha^{i}, p_{i}(t) \in \mathbb{K}[t]$. Also, note 
that both $M(t)$ and the denominator of $u(t)$ are monic, and hence $l_{r-1}=1$. First of all, we prove that there are exactly $r$ polynomials in $\left\{p_{i}(t), i=0, \ldots, n-1\right\} \subset \mathbb{K}[t]$ which are linearly independent. For this purpose we observe that the coefficients of $m(t),\left\{1, l_{r-2}, \ldots, l_{0}\right\} \subset \mathbb{L}$ are linearly independent over $\mathbb{K}$. Indeed, from the equality $M(t)=(t+d) m(t)$, one has that $l_{r-i}=(-d)^{i-1}+(-d)^{i-2} k_{r-1}+\cdots+$ $k_{r-i+1}$, for $i=2, \ldots, r$. So, $\left\{1, l_{r-2}, \ldots, l_{0}\right\} \subset \mathbb{L}$ are $\mathbb{K}$-linearly independent, since otherwise one would find a non-zero polynomial of degree smaller than $r$ vanishing at $-d$. Now, let $\vec{l}_{i}=\left(l_{i, 0}, \ldots, l_{i, n-1}\right)^{t}$ be the vector of coordinates of $l_{i}$ in the base $\left\{1, \alpha, \ldots, \alpha^{n-1}\right\}$. Then, $\left\{\overrightarrow{1}, \vec{l}_{r-2}, \ldots, \vec{l}_{0}\right\} \subset \mathbb{K}^{n}$ are $\mathbb{K}$-linearly independent. Moreover, since $\left(p_{0}(t), \ldots, p_{n-1}(t)\right)^{t}=\overrightarrow{1} t^{r-1}+\vec{l}_{r-2} t^{r-2}+\cdots+\vec{l}_{0}$, there are $r$ polynomials $p_{i_{j}}, 0 \leq i_{1}<\cdots<i_{r} \leq n-1$, linearly independent. By simplicity, we assume w.l.o.g. that the first $r$ polynomials are linearly independent. Observe that this is always possible through a permutation matrix. The new curve, that we will continue denoting by $\mathcal{U}$, is not, in general, a hypercircle. In this situation, we proceed to prove (1) and (2).

In order to prove (1), let $\mathcal{A} \in \mathcal{M}_{n-r \times r}(\mathbb{K})$ be the matrix providing the linear combinations of the $n-r$ last polynomials in terms of the first $r$ polynomials; i.e., $\left(p_{r}(t), \ldots, p_{n-1}(t)\right)^{t}=\mathcal{A}\left(p_{0}(t), \ldots, p_{r-1}(t)\right)^{t}$. Now, given the bases $\mathcal{B}=\left\{1, \ldots, t^{r-1}\right\}$ and $\mathcal{B}^{\star}=\left\{p_{0}(t), \ldots, p_{r-1}(t)\right\}$, let $\mathcal{M} \in \mathcal{M}_{r \times r}(\mathbb{K})$ be the transpose matrix of change of bases from $\mathcal{B}$ to $\mathcal{B}^{\star}$. Finally, the $n \times n$ matrix

$$
\mathcal{Q}=\left(\begin{array}{cc}
\mathcal{M} & \mathcal{O}_{r, n-r} \\
-\mathcal{A} & I_{n-r}
\end{array}\right)
$$

defines, under the previous assumptions, the affine transformation $\chi$. Note that if $r=n$, then $\mathcal{Q}=\mathcal{M}$.

The proof of (2) is analogous to (1). Now let us consider the bases $\mathcal{B}=\left\{1, \ldots, t^{r-1}, t^{r}\right\}$ and $\mathcal{B}^{\star}=\left\{p_{0}(t), \ldots, p_{r-1}(t), M(t)\right\}$. Let $\mathcal{A} \in \mathcal{M}_{n-r \times r+1}(\mathbb{K})$ be the matrix providing the linear combinations of the $n-r$ last polynomials in terms of basis $\mathcal{B}^{\star}$; i.e., $\left(p_{r}(t), \ldots, p_{n-1}(t)\right)^{t}=\mathcal{A}\left(p_{0}(t), \ldots, p_{r-1}(t), M(t)\right)^{t}$. Let $\mathcal{M} \in \mathcal{M}_{r+1 \times r+1}(\mathbb{K})$ be the transpose matrix of change of basis from $\mathcal{B}$ to $\mathcal{B}^{\star}$. Finally, the $n+1 \times n+1$ matrix

$$
\mathcal{Q}=\left(\begin{array}{cc}
\mathcal{M} & \mathcal{O}_{r+1, n-r} \\
-\mathcal{A} & I_{n-r}
\end{array}\right)
$$

defines, under the previous assumptions, the projective transformation $\rho$. Note that if $r=n$, then $\mathcal{Q}=\mathcal{M}$.

As a direct consequence, we derive the following geometric properties of hypercircles.

Corollary 3.2. In the hypothesis of Theorem 3.1;

(1) $\mathcal{U}$ defines a curve of degree $r$.

(2) $\mathcal{U}$ is contained in a linear variety of dimension $r$ and it is not contained in a variety of dimension $r-1$.

(3) $\mathcal{U}$ is a regular curve in $\mathbb{P}(\mathbb{F})^{n}$.

(4) The Hilbert function of $\mathcal{U}$ is equal to its Hilbert polynomial and $h_{\mathcal{U}}(m)=$ $m r+1$.

Proof. All of these properties are well known to hold for the rational normal curve of degree $r$ (e.g. [6], 7], 18]). 
In the following theorem, we classify the hypercircles that are affinely equivalent over $\mathbb{K}$. We will assume that the denominator of the generating units are not constant. The case where the units are polynomials are described in Theorem 2.6.

Theorem 3.3. Let $\mathcal{U}_{i}, i=1,2$, be $\alpha$-hypercircles associated to $u_{i}(t)=\frac{a_{i} t+b_{i}}{t+d_{i}}$, and let $M_{i}(t)$ be the minimal polynomial of $-d_{i}$ over $\mathbb{K}$. Then, the following statements are equivalent:

(1) $\mathcal{U}_{1}$ and $\mathcal{U}_{2}$ are affinely equivalent over $\mathbb{K}$.

(2) There exists a unit $\tau(t) \in \mathbb{K}(t)$ such that it maps a root (and hence all roots) of $M_{1}(t)$ onto a root (resp. all roots) of $M_{2}(t)$.

Proof. First of all, note that, because of Theorem 2.6, the result for lines is trivial. For dealing with the general case, we observe that, by Lemma 2.5 we can assume that $u_{i}(t)=1 /\left(t+d_{i}\right)$. Next, suppose that $\mathcal{U}_{1}$ and $\mathcal{U}_{2}$ are affinely equivalent over $\mathbb{K}$. By Theorem [3.1, statement $(1),\left[\mathbb{K}\left(d_{1}\right): \mathbb{K}\right]=\left[\mathbb{K}\left(d_{2}\right): \mathbb{K}\right]=r$ and the curves $\mathcal{U}_{1}^{\star}:=\chi\left(\mathcal{U}_{1}\right)$ and $\mathcal{U}_{2}^{\star}:=\chi\left(\mathcal{U}_{2}\right)$ parametrized by $\tilde{\chi}_{1}(t)=\left(\frac{1}{M_{1}(t)}, \ldots, \frac{t^{r-1}}{M_{1}(t)}\right)$ and $\tilde{\chi}_{2}(t)=\left(\frac{1}{M_{2}(t)}, \ldots, \frac{t^{r-1}}{M_{2}(t)}\right)$, respectively, are affinely equivalent over $\mathbb{K}$; note that, for simplicity we have omitted the last zero components in these parametrizations. Therefore, there exists $\mathcal{A}=\left(a_{i, j}\right) \in G L(r, \mathbb{K})$ and $\vec{v} \in M_{r \times 1}(\mathbb{K})$, such that $\varphi(t):=$ $\mathcal{A} \tilde{\chi}_{1}(t)^{t}+\vec{v}$ parametrizes $\mathcal{U}_{2}^{\star}$. In consequence, since $\varphi(t)$ and $\tilde{\chi}_{2}(t)$ are proper parametrizations of the same curve, there exists a unit $\tau(t) \in \mathbb{K}(t)$ such that $\varphi(t)=$ $\tilde{\chi}_{2}(\tau(t))$. Then, considering the first component in the above equality, one gets that

$$
\left(a_{1,1}+\cdots+a_{1, r} t^{r-1}+v_{1} M_{1}(t)\right) M_{2}(\tau(t))=M_{1}(t) .
$$

Now, substituting $t$ by $-d_{1}$, we obtain

$$
\left(a_{1,1}+\cdots+a_{1, r}\left(-d_{1}\right)^{r-1}+v_{1} M_{1}\left(-d_{1}\right)\right) M_{2}\left(\tau\left(-d_{1}\right)\right)=M_{1}\left(-d_{1}\right)=0 .
$$

Note that $a_{1,1}+\cdots+a_{1, r}\left(-d_{1}\right)^{r-1} \neq 0$, because $\left[\mathbb{K}\left(d_{1}\right): \mathbb{K}\right]=r$. Also, note that $\tau\left(-d_{1}\right)$ is well defined, because $-d_{1}$ does not belong to $\mathbb{K}$. This implies that $M_{2}\left(\tau\left(-d_{1}\right)\right)=0$. So, $\tau\left(-d_{1}\right)$ is a root of $M_{2}(t)$.

Conversely, let $\tau(t)=\frac{k_{1} t+k_{2}}{k_{3} t+k_{4}} \in \mathbb{K}(t)$ be a unit that maps the root $\gamma$ of $M_{1}(t)$ onto the root $\beta$ of $M_{2}(t)$, i.e., $\tau(\gamma)=\beta$. This relation implies that $\mathbb{K}(\gamma)=\mathbb{K}(\beta)$ and that $\operatorname{deg}\left(M_{1}(t)\right)=\operatorname{deg}\left(M_{2}(t)\right)=r$. Therefore, because of Theorem 3.1, it is enough to prove that the curves $\mathcal{U}_{1}^{\star}:=\chi\left(\mathcal{U}_{1}\right)$ and $\mathcal{U}_{2}^{\star}:=\chi\left(\mathcal{U}_{2}\right)$ are affinely equivalent over $\mathbb{K}$. Recall that $\mathcal{U}_{i}^{\star}$ is parametrized by $\varphi_{i}(t):=\tilde{\chi}(t)=\left(\frac{1}{M_{i}(t)}, \ldots, \frac{t^{r-1}}{M_{i}(t)}\right)$; here again, we omit the last zero components of the parametrization. In order to prove the result, we find an invertible matrix $\mathcal{A} \in G L(r, \mathbb{K})$ and a vector $\vec{v} \in M_{r \times 1}(\mathbb{K})$, such that $\mathcal{A} \varphi_{1}^{t}(t)+\vec{v}=\varphi_{2}^{t}(\tau(t))$. For this purpose, we consider the polynomial $M(t)=M_{2}(\tau(t))\left(k_{3} t+k_{4}\right)^{r} \in \mathbb{K}[t]$. Now, since $\tau(t)$ is a unit of $\mathbb{K}(t)$, and the roots of $M_{2}(t)$ are not in $\mathbb{K}$, one gets that $\operatorname{deg}(M)=\operatorname{deg}\left(M_{2}\right)=r$. Moreover, since $\gamma$ is a root of $M(t)$, and taking into account that $M_{1}(t)$ is the minimal polynomial of $\gamma$ over $\mathbb{K}$ and that $\operatorname{deg}(M)=r=\operatorname{deg}\left(M_{1}\right)$, one has that there exists $c \in \mathbb{K}^{*}$ such that $M(t)=c M_{1}(t)$. Now, in order to determine $\mathcal{A}$ and $\vec{v}$, let us substitute $\tau(t)$ in the $i$-th component of $\varphi_{2}(t)$ :

$$
\frac{\tau(t)^{i}}{M_{2}(\tau(t))}=\frac{\tau(t)^{i}\left(k_{3} t+k_{4}\right)^{r}}{M_{2}(\tau(t))\left(k_{3} t+k_{4}\right)^{r}}=\frac{\left(k_{1} t+k_{2}\right)^{i}\left(k_{3} t+k_{4}\right)^{r-i}}{c M_{1}(t)} .
$$


Since the numerator and the denominator in the above rational function have the same degree, taking quotients and remainders, $\varphi_{2}(t)$ can be expressed as

$$
\left(\varphi_{2}(\tau(t))\right)_{i=1, \ldots, r}=\left(v_{i}+\frac{a_{i, 1}+\cdots+a_{i, r} t^{r-1}}{M_{1}(t)}\right)_{i=1, \ldots, r},
$$

for some $v_{i}, a_{i, j} \in \mathbb{K}$. Take $\mathcal{A}=\left(a_{i, j}\right)$ and $\vec{v}=\left(v_{i}\right)$. Then, $\mathcal{A}\left(\varphi_{1}(t)\right)^{t}+\vec{v}=$ $\left(\varphi_{2}(\tau(t))^{t}\right.$. Finally, let us see that $\mathcal{A}$ is regular. Indeed, suppose that $\mathcal{A}$ is singular and that there exists a nontrivial linear relation $\lambda_{1} F_{1}+\cdots+\lambda_{r} F_{r}=\overrightarrow{0}$, where $F_{i}$ denotes the $i$-th row of $\mathcal{A}$. This implies that $\left(\lambda_{1} \frac{1}{M_{2}(t)}+\cdots+\lambda_{r} \frac{t^{r-1}}{M_{2}(t)}\right) \circ \tau(t)=$ $\lambda_{1} v_{1}+\cdots+\lambda_{r} v_{r}$ is constant, which is impossible because $\frac{\lambda_{1}+\cdots+\lambda_{r} t^{r-1}}{M_{2}(t)}$ is not constant and $\tau(t)$ is a unit of $\mathbb{K}(t)$.

For two true circles, there is always a real affine transformation relating them. Note that this is not the case for hypercircles. For example, consider $\mathbb{K}=\mathbb{Q}$ and $\alpha$ a root of $x^{4}+x^{3}+1$, then the hypercircles associated to the units $\frac{1}{t+\alpha}$ and $\frac{1}{t+\alpha^{2}}$ are not affinely equivalent over $\mathbb{Q}$. However, for algebraic extensions of degree 2 (where the circle case fits) and of degree 3 , we recover this property for hypercircles that are not lines.

Corollary 3.4. Let $\mathbb{K}(\alpha)$ be an extension of degree 2 or 3 . Then all $\alpha$-hypercircles, that are not lines, are affinely equivalent over $\mathbb{K}$.

Proof. By Lemma 2.5, we may assume that the hypercircles are associated to units of the form $\frac{1}{t+d}$. Now, we consider two $\alpha$-hypercircles not being lines, namely, let $\mathcal{U}_{i}$ be the $\alpha$-hypercircle associated to $\frac{1}{t+d_{i}}$ for $i=1,2$, and $d_{i} \notin \mathbb{K}$.

If the extension is of degree 2 , let $d_{i}=\lambda_{i}+\mu_{i} \alpha$, with $\lambda_{i}, \mu_{i} \in \mathbb{K}$ and $\mu_{i} \neq 0$. Then, the unit $\tau(t)=\tau_{0}+\tau_{1} t \in \mathbb{K}[t]$ where $\tau_{0}=\frac{\mu_{2} \lambda_{1}-\mu_{1} \lambda_{2}}{\mu_{1}}$ and $\tau_{1}=\frac{\mu_{2}}{\mu_{1}}$, verifies that $\tau\left(-d_{1}\right)=-d_{2}$. By Theorem $3.3, \mathcal{U}_{1}$ and $\mathcal{U}_{2}$ are affinely equivalent over $\mathbb{K}$.

If the extension is of degree 3 , let $x^{3}+m x^{2}+n x+r$ be the minimal polynomial of $\alpha$, and let $d_{i}=\lambda_{i}+\mu_{i} \alpha+\eta_{i} \alpha^{2}$, if $\eta_{i}=0$, then $d_{i}$ is trivially the image of $\alpha$ by the unit $\lambda_{i}+\mu_{i} t$. If $\eta_{i} \neq 0$, then $d_{i}$ is the image of $\alpha$ by the unit $\tau_{i}(t)=\frac{\tau_{i, 1} t+\tau_{i, 0}}{\eta_{i} t+\tau_{i, 2} t}$, where $\tau_{i, 0}=-\lambda_{i} \mu_{i}+\lambda_{i} m \eta_{i}-r \eta_{i}^{2}, \tau_{i, 1}=-n \eta_{i, 2}+\lambda_{i} \eta_{i}-\mu_{i}^{2}+\mu_{i} \eta_{i} m, \tau_{i, 2}=-\mu_{i}+m \eta_{i}$. This is indeed a unit, since, if not, $d_{i}$ would belong to $\mathbb{K}$. Hence $d_{i}$ is the image of $\alpha$ by the unit $\tau_{i}$. It follows that $d_{2}$ is the image of $d_{1}$ by the unit $\tau_{2} \circ \tau_{1}$ and both hypercircles are affinely equivalent over $\mathbb{K}$.

In Corollary 3.2 we have seen that the degree of a hypercircle is given by the degree of the field extension provided by the pole of any nonpolynomial generating unit. Lines are curves of degree one, a particular case of this phenomenon. Now, we consider other kind of hypercircles of degree smaller than $n$. This motivates the following concept.

Definition 3.5. Let $\mathcal{U}$ be an $\alpha$-hypercircle. If the degree of $\mathcal{U}$ is $[\mathbb{K}(\alpha): \mathbb{K}]$, we say that it is a primitive hypercircle. Otherwise, we say that $\mathcal{U}$ is a nonprimitive hypercircle.

Regarding the complex numbers as an extension of the reals, lines may be considered as circles when we define them through a Moebius transformation. Lines are the only one curves among these such that its degree is not $[\mathbb{C}: \mathbb{R}]$. The situation is more complicated in the general case. Apart from lines, which have been 
thoroughly studied in Theorem 2.6, there are other nonprimitive hypercircles. This is not a big challenge because, as we will see, nonprimitive hypercircles are primitive on another extension. Moreover, these cases reflect some algebraic aspects of the extension $\mathbb{K} \subseteq \mathbb{K}(\alpha)=\mathbb{L}$ in the geometry of the hypercircles. Actually, we will see that there is a correspondence between nonprimitive hypercircles and the intermediate fields of $\mathbb{K} \subseteq \mathbb{L}$. More precisely, let $\mathcal{U}$ be a nonprimitive hypercircle associated to $u(t)=\frac{1}{t+d}$, where $r=[\mathbb{K}(d): \mathbb{K}]<[\mathbb{L}: \mathbb{K}]=n$. In this case, we have the algebraic extensions $\mathbb{K} \subseteq \mathbb{K}(d) \subsetneq \mathbb{L}$. We may consider $u(t)$ as a unit either in the extension $\mathbb{K} \subseteq \mathbb{K}(d)$ with primitive element $d$ or in $\mathbb{K}(d) \subsetneq \mathbb{L}$ with primitive element $\alpha$. In the first case, $u(t)$ defines a primitive hypercircle in $\mathbb{F}^{r}$. In the second case, as $u(t)$ is a $\mathbb{K}(d)$ unit, it defines a line. The analysis of $\mathcal{U}$ can be reduced to the case of the primitive hypercircle associated to $u(t)$ in the extension $\mathbb{K} \subseteq \mathbb{K}(d)$.

Theorem 3.6. Let $\mathcal{U}$ be the nonprimitive hypercircle associated to $u(t)=\frac{a t+b}{t+d} \in$ $\mathbb{K}(\alpha)(t)$. Let $\mathcal{V}$ be the hypercircle generated by the unit $\frac{1}{t+d}$ in the extension $\mathbb{K} \subseteq$ $\mathbb{K}(d)$. Then, there is an affine inclusion from $\mathbb{F}^{r}$ to $\mathbb{F}^{n}$, defined over $\mathbb{K}$, that maps the hypercircle $\mathcal{V}$ onto $\mathcal{U}$.

Proof. Taking into account Lemma 2.5] we may assume that $u(t)=\frac{1}{t+d}$. Let $\phi(t)=\left(\phi_{0}(t), \ldots, \phi_{n-1}(t)\right) \in \mathbb{K}(t)^{n}$ be the parametrization of $\mathcal{U}$, obtained from $u(t)$, with respect to the basis $\mathcal{B}=\left\{1, \alpha, \ldots, \alpha^{n-1}\right\}$. Similarly, let $\psi(t)=\left(\psi_{0}(t)\right.$, $\left.\ldots, \psi_{r-1}(t)\right) \in \mathbb{K}^{r}(t)$ be the parametrization of the hypercircle $\mathcal{V}$, associated to $u(t)$, with respect to the basis $\mathcal{B}^{\star}=\left\{1, d, \ldots, d^{r-1}\right\}$, where $r=[\mathbb{K}(d): \mathbb{K}]$. The matrix $\mathcal{D}=\left(d_{j i}\right) \in \mathcal{M}_{n \times r}(\mathbb{K})$ whose columns are the coordinates of $d^{i}$ with respect to $\mathcal{B}$ induces a $\mathbb{K}$-linear transformation $\chi: \mathbb{F}^{r} \mapsto \mathbb{F}^{n}$ that maps $\mathcal{V}$ onto $\mathcal{U}$. Indeed, as $u(t)=\sum_{i=0}^{r-1} \psi_{i}(t) d^{i}=\sum_{j=0}^{n-1} \phi_{j}(t) \alpha^{j}$, one has that

$$
\sum_{i=0}^{r-1} \psi_{i}(t) d^{i}=\sum_{i=0}^{r-1} \psi_{i}(t)\left(\sum_{j=0}^{n-1} d_{j, i} \alpha^{j}\right)=\sum_{j=0}^{n-1}\left(\sum_{i=0}^{r-1} d_{j, i} \psi_{i}(t)\right) \alpha^{j}=\sum_{j=0}^{n-1} \phi_{j}(t) \alpha^{j} .
$$

Then $\phi(t)^{t}=\mathcal{D} \psi(t)^{t}$. Moreover, $\chi$ is one-to-one, because $\operatorname{rank}(D)=r$.

As a consequence of this theorem, every hypercircle is affinely equivalent, over $\mathbb{K}$, to a primitive hypercircle. Therefore, the study of hypercircles can be reduced to the study of primitives hypercircles.

\section{Properties at infinity of a hypercircle}

Circles have a very particular structure at infinity, namely, they pass through the cyclic points, i.e., $[ \pm i: 1: 0]$, which are related to the minimal polynomial defining the circle as a hypercircle as remarked in the introduction. In this section, we will see that a similar situation occurs for more general primitive hypercircles. More precisely, let $\mathcal{U}$ be the primitive hypercircle defined by the unit $u(t)=\frac{a t+b}{t+d}$. By Corollary [3.2, $\mathcal{U}$ is a parametric affine curve of degree $n$. So, there are at most $n$ different points in the hyperplane at infinity. Let $\phi(t)=\left(\phi_{0}(t), \ldots, \phi_{n-1}(t)\right)$ be the parametrization of $\mathcal{U}$ generated by $u(t)$; recall that $\phi_{i}(t)=\frac{p_{i}(t)}{M(t)}$. Thus, projective coordinates of the points attained by $\phi(t)$ are given by $\left[p_{0}(t): \cdots: p_{n-1}(t): M(t)\right]$. Now, substituting $t$ by every conjugate $\sigma(-d)$ of $-d$, we obtain

$$
\left[p_{0}(\sigma(-d)): \cdots: p_{n-1}(\sigma(-d)): 0\right]=\left[\sigma\left(p_{0}(-d)\right): \cdots: \sigma\left(p_{n-1}(-d)\right): 0\right] .
$$

We prove next that these points are the points of the hypercircle at infinity. 
Lemma 4.1. Let $\mathcal{U}$ be a primitive hypercircle associated to the unit $u(t)=\frac{a t+b}{t+d}$. The $n$ points at infinity are

$$
P_{j}=\left[\sigma_{j}\left(p_{0}(-d)\right): \cdots: \sigma_{j}\left(p_{n-1}(-d)\right): 0\right], 1 \leq j \leq n
$$

where $\sigma_{j}$ are the $\mathbb{K}$-automorphisms of the normal closure of $\mathbb{L}=\mathbb{K}(\alpha)$ over $\mathbb{K}$.

Proof. First of all, observe that $\operatorname{gcd}\left(p_{0}, \ldots, p_{n-1}, M\right)=1$, and hence $P_{j}$ are well defined. Moreover, $p_{i}(-d) \neq 0$, for every $i \in\{0, \ldots, n-1\}$, since $p_{i}(t) \in \mathbb{K}[t]$ is of degree at most $n$ and, thus, if $p_{i}(-d)=0$, then $\frac{p_{i}(t)}{M(t)}=c \in \mathbb{K}$ and the hypercircle would be contained in a hyperplane. But this is impossible since $\mathcal{U}$ is primitive (see Corollary 3.2). It remains to prove that they are different points. Suppose that two different tuples define the same projective point. We may suppose that $P_{1}=P_{j} . P_{1}$ verifies that $\sum_{i=0}^{n-1} p_{i}(-d) \alpha^{i}=(-a d+b) m(-d) \neq 0$ and $P_{j}$ verifies that $\sum_{i=0}^{n-1} p_{i}\left(\sigma_{j}(-d)\right) \alpha^{i}=\left(a \sigma_{j}(-d)+b\right) m\left(\sigma_{j}(-d)\right)=0$. Thus, $P_{j}$ is contained in the projective hyperplane $\sum_{i=0}^{n-1} \alpha^{i} X_{i}=0$, but not in $P_{1}$. Hence, $P_{1} \neq P_{j}$.

Let us check that, as in the case of circles, the points at infinity of primitive $\alpha$-hypercircles do not depend on a particular hypercircle.

Theorem 4.2. For a fixed extension $\mathbb{K} \subseteq \mathbb{K}(\alpha)$ of degree $n$, the set of points at the infinity $P=\left\{P_{1}, \ldots, P_{n}\right\}$ of any primitive hypercircle does not depend on the particular $\alpha$-hypercircle $\mathcal{U}$, but only on the algebraic extension and on the primitive element $\alpha$. Moreover, the set $P$ is characterized by the following property:

$$
\left\{X_{0}+\alpha_{j} X_{1}+\cdots+\alpha_{j}^{n-1} X_{n-1}=0\right\} \cap \overline{\mathcal{U}} \cap\left\{X_{n}=0\right\}=P \backslash\left\{P_{j}\right\},
$$

where $\alpha_{j}=\sigma_{j}(\alpha)$ are the conjugates of $\alpha$ in $\mathbb{F}, 1 \leq j \leq n, \overline{\mathcal{U}}$ is the projective closure of $\mathcal{U}$ and $\left\{X_{n}=0\right\}$ is the hyperplane at infinity.

Proof. Let $\mathcal{U}$ be the primitive $\alpha$-hypercircle generated by a unit $u(t)=\frac{a t+b}{t+d} \cdot \overline{\mathcal{U}}$ has the projective parametrization $\left[p_{0}(t): \cdots: p_{n-1}(t): M(t)\right]$. Let $P_{j}=\left[\sigma_{j}\left(p_{0}(-d)\right)\right.$ : $\left.\cdots: \sigma_{j}\left(p_{n-1}(-d)\right): 0\right]$. Its evaluation in the equation of hyperplane $X_{0}+\alpha_{k} X_{1}+$ $\ldots+\alpha_{k}^{n-1} X_{n-1}$, yields:

$$
\begin{gathered}
\sum_{i=0}^{n-1} \sigma_{j}\left(p_{i}(-d)\right) \alpha_{k}^{i}=\sigma_{k}\left(\sum_{i=0}^{n-1} \sigma_{k}^{-1} \circ \sigma_{j}\left(p_{i}(-d)\right) \alpha^{i}\right) \\
=\sigma_{k}\left(\left(a\left(\sigma_{k}^{-1} \circ \sigma_{j}(-d)\right)+b\right) m\left(\sigma_{k}^{-1} \circ \sigma_{j}(-d)\right)\right) .
\end{gathered}
$$

If $j=k$, the previous expression equals $\sigma_{k}((-a d+b) m(-d)) \neq 0$. If $j \neq k$, then $\sigma_{k}^{-1} \circ \sigma_{j}(-d)$ is a conjugate of $-d$, different from $-d$, because $-d$ is a primitive element. So $m\left(\sigma_{k}^{-1} \circ \sigma_{j}(-d)\right)=0$.

In order to show that this point does not depend on a particular hypercircle, take the $n$ hyperplanes $X_{0}+\alpha_{k} X_{1}+\cdots+\alpha_{k}^{n-1} X_{n-1}=0, k=1 \ldots n$. Every point at infinity of a hypercircle is contained in exactly $n-1$ of those hyperplanes. Also, any of these hyperplanes contains exactly $n-1$ points at infinity of the hypercircle. One point at infinity may be computed by solving the linear system given by any combination of $n-1$ hyperplanes. The matrix of the linear system is a Vandermonde matrix, each row depending on the corresponding $\alpha_{k}$, so there is only one solution.

Remark 4.3. Notice that this theorem provides an $n$-simplex combinatorial structure of the points at infinity of any primitive hypercircle. 
The following result shows that the points at infinity can be read directly from the minimal polynomial of $\alpha$.

Proposition 4.4. Let $M_{\alpha}(t)$ be the minimal polynomial of $\alpha$ over $\mathbb{K}$. Let $m_{\alpha}(t)=$ $\frac{M_{\alpha}(t)}{t-\alpha}=\sum_{i=0}^{n-1} l_{i} t^{i} \in \mathbb{K}(\alpha)[t]$, where $l_{n-1}=1$. Then, the points at infinity of every primitive $\alpha$-hypercircle are $\left[l_{0}: l_{1}: \cdots: l_{n-2}: l_{n-1}: 0\right]$ and its conjugates.

Proof. We consider the symmetric polynomial $r(x, y)=\frac{M_{\alpha}(x)-M_{\alpha}(y)}{x-y}$. Substituting $(x, y)$ by $(t, \alpha)$ we obtain that

$$
r(t, \alpha)=\frac{M_{\alpha}(t)-M_{\alpha}(\alpha)}{t-\alpha}=\frac{M_{\alpha}(t)}{t-\alpha}=m_{\alpha}(t) .
$$

That is, $m_{\alpha}(t)$ is symmetric in $t$ and $\alpha$. Now take the hypercircle induced by the unit $\frac{1}{t-\alpha}=\frac{m_{\alpha}(t)}{M_{\alpha}(t)}$. By Lemma 4.1, we already know that one point at infinity is $\left[p_{0}(\alpha)\right.$ : $\left.\cdots: p_{n-1}(\alpha): 0\right]$, where $m_{\alpha}(t)=\sum_{i=0}^{n-1} p_{i}(t) \alpha^{i}$. By symmetry, $\sum_{i=0}^{n-1} p_{i}(t) \alpha^{i}=$ $\sum_{i=0}^{n-1} p_{i}(\alpha) t^{i}$. That is, $p_{i}(\alpha)=l_{i}$. Thus, the points at infinity are $\left[l_{0}: l_{1}: \cdots\right.$ : $\left.l_{n-2}: 1: 0\right]$ and its conjugates.

The next result deals with the tangents of a hypercircle at infinity, and it explains again why parabolas cannot be hypercircles.

Proposition 4.5. The tangents to a primitive hypercircle at the points at infinity are not contained in the hyperplane at infinity.

Proof. Let $\mathcal{U}$ be the primitive $\alpha$-hypercircle generated by $\frac{a t+b}{t+d}$, and $\left[p_{0}(t): \cdots\right.$ : $\left.p_{n-1}(t): M(t)\right]$ the projective parametrization generated by the unit. In the proof of Lemma 4.1, we have seen that $p_{n-1}(t)$ is not identically 0 , because $p_{n-1}(-d) \neq 0$. So, we can dehomogenize w.r.t. the variable $X_{n-1}$, obtaining the affine parametrization $\left(\frac{p_{0}(t)}{p_{n-1}(t)}, \ldots, \frac{p_{n-2}(t)}{p_{n-1}(t)}, \frac{M(t)}{p_{n-1}(t)}\right)$ of $\mathcal{U}$ on another affine chart. We have to check that the tangents to the curve at the intersection points with the hyperplane $X_{n-1}=0$ are not contained in this hyperplane. The points of $\mathcal{C}$ in the hyperplane $X_{n-1}=0$ are obtained by substituting $t$ by $\sigma(-d)$. The last coordinate of the tangent vector is

$$
\frac{M^{\prime}(t) p_{n-1}(t)-M(t) p_{n-1}^{\prime}(t)}{p_{n-1}(t)^{2}}
$$

We evaluate this expression at $\sigma(-d) . M(\sigma(-d))=0$ and, as all of its roots are different in $\mathbb{F}, M^{\prime}(\sigma(-d)) \neq 0$. We also know that $\sigma\left(p_{n-1}(-d)\right) \neq 0$. Hence, the last coordinate of the tangent vector is nonzero. Thus, the tangent line is not contained in the hyperplane at infinity.

Finally, we present a property of hypercircles that can be derived from the knowledge of its behavior at infinity. We recall a property of circles stating that given three different points in the plane, there is exactly one circle passing through them (which is a line if they are collinear). The result is straightforward if we recall that there is only one conic passing throught five points. In the case of circles, we have the two points at infinity already fixed, so, given three points in the affine plane there will only be a conic (indeed a circle if it passes through the cyclic points at infinity) through them. Even if hypercircles are curves in $n$-space, surprisingly, the same occurs for hypercircles.

We are going to prove that, given 3 different points in $\mathbb{K}^{n}$, there is exactly one hypercircle passing through them. If the points are not in general position, the 
resulting hypercircle need not be a primitive one. First, we need a lemma that states what the points over $\mathbb{K}$ are of the hypercircle that are reachable by the parametrization.

Lemma 4.6. Let $\mathcal{U}$ be the $\alpha$-hypercircle, not necessarily primitive, associated to $u(t)=\frac{a t+b}{t+d}$ with induced parametrization $\Phi(t) . \Phi(\mathbb{K})=\mathcal{U} \cap \mathbb{K}^{n} \backslash\{\bar{a}\}$ with $a=$ $\sum_{i=0}^{n-1} a_{i} \alpha^{i}, \bar{a}=\left(a_{0}, \ldots, a_{n-1}\right)$.

Proof. We already know that $\Phi(t)$ is proper and, obviously, $\Phi(\mathbb{K}) \subseteq \mathcal{U} \cap \mathbb{K}^{n}$, also, $\bar{a}$ is not reachable by $\Phi(t)$, since otherwise one would have that $a=u(\lambda)$ for some $\lambda$, and this implies that $a d-b=0$, which is impossible since $u(t)$ is a unit. In order to prove the other inclusion, write as before $\phi_{i}(t)=\frac{p_{i}(t)}{M(t)}$, where $M(t)$ is the minimal polynomial of $-d$ over $\mathbb{K}$. Then, we consider the ideal $I$ over $\mathbb{F}[t, \bar{X}]$ generated by $\left(p_{0}(t)-X_{0} M(t), \ldots, p_{n-1}(t)-X_{n-1} M(t)\right)$, where $\bar{X}=\left(X_{0}, \ldots, X_{n-1}\right)$, and the ideal $J=I+(Z M(t)-1) \subseteq \mathbb{F}[Z, t, \bar{X}]$. Let $I_{1}$ be the first elimination ideal of $I$; i.e., $I_{1}=I \cap \mathbb{F}[\bar{X}]$ and let $J_{2}$ be the second elimination ideal of $J$; i.e., $J_{2}=J \cap \mathbb{F}[\bar{X}]$. Observe that $I \subseteq J$ and therefore $I_{1} \subseteq J_{2}$. Note that $\mathcal{U}=V\left(J_{2}\right)$; i.e., $\mathcal{U}$ is the variety defined by $J_{2}$ over $\mathbb{F}$. Thus $\mathcal{U} \subseteq V\left(I_{1}\right)$. Now, let us take $\bar{x} \in\left(\mathcal{U} \cap \mathbb{K}^{n}\right) \backslash\{\bar{a}\}$. Then $\bar{x} \in V\left(I_{1}\right)$. Observe that, by construction, the leading coefficient of $p_{i}(t)-X_{i} M(t)$ w.r.t. $t$ is $a_{i}-X_{i}$. Therefore, since $\bar{x} \neq \bar{a}$ one has that at least one of the leading coefficients of the polynomials in $I$ w.r.t. $t$ does not vanish at $\bar{x}$. Thus, applying the Extension Theorem (see Theorem 3, p. 117 in [5]), there exists $t_{0} \in \mathbb{F}$ such that $\left(t_{0}, \bar{x}\right) \in V(I)$. This implies that $p_{i}\left(t_{0}\right)-x_{i} M\left(t_{0}\right)=0$ for $i=1 \ldots n-1$. Let us see that $M\left(t_{0}\right) \neq 0$. Indeed, if $M\left(t_{0}\right)=0$, then $p_{i}\left(t_{0}\right)$ is also zero for every index and therefore $\operatorname{gcd}\left(p_{0}(t), \ldots, p_{n-1}(t), M(t)\right) \neq 1$, which is impossible. Hence $\Phi$ is defined at $t_{0}$ and $\Phi\left(t_{0}\right)=\bar{x}$. To conclude, we only need to show that $t_{0} \in \mathbb{K}$. For this purpose, we note that the inverse of $\Phi(t)$ is given by

$$
P(\bar{X})=\frac{-d \sum X_{i} \alpha^{i}+b}{\sum X_{i} \alpha^{i}-a} .
$$

Now, since $\bar{x} \neq \bar{a}$, one deduces that $P(\bar{x})$ is well defined, and the only parameter value generating $\bar{x}$ is $t_{0}=P(\bar{x})$. Hence, the gcd of the polynomials $p_{i}(t)-x_{i} M(t)$ is a power of $\left(t-t_{0}\right)$. Thus, taking into account that $p_{i}, M \in \mathbb{K}[t]$, one deduces that $t_{0} \in \mathbb{K}$. Finally, it only remains to state that $\bar{a}$ is generated when $t$ takes the value of the infinity of $\mathbb{K}$. But this follows taking $\Phi(1 / t)$ and substituting by $t=0$.

Proposition 4.7. Let $X_{i}=\left(X_{i 0}, \ldots, X_{i, n-1}\right) \in \mathbb{K}^{n} \subseteq \mathbb{F}^{n}, 1 \leq i \leq 3$ be three different points. Then, there exists only one $\alpha$-hypercircle passing through them.

Proof. Let $Y_{i}=\sum_{j=0}^{n-1} X_{i j} \alpha^{j} \in \mathbb{K}(\alpha), 1 \leq i \leq 3$. Consider the following linear homogeneous system in $a, b, c, d$ :

$$
b=Y_{1} d, a+b=Y_{2}(c+d), a=Y_{3} c .
$$

Observe that, if the three points are different, there is only one projective solution, namely $[a: b: c: d]$ where $a=Y_{1} Y_{3}-Y_{3} Y_{2}, b=Y_{1} Y_{2}-Y_{1} Y_{3}, c=Y_{1}-Y_{2}$, $d=Y_{2}-Y_{3}$.

Take the unit $u(t)=\frac{a t+b}{c t+d}$. It verifies that $u(0)=Y_{1}, u(1)=Y_{2}, u(\infty)=Y_{3}$. Then, the hypercircle associated to $u$ passes through $X_{1}, X_{2}, X_{3}$. In order to prove that this hypercircle is unique, let $v$ be the unit associated to a hypercircle passing through the three points and $\psi(t)$ the parametrization induced by $v(t)$. By Lemma 
4.6. as $X_{i} \in \mathbb{K}^{n}$, the point $X_{i}$ is reached for a parameter value $t_{i}$ in $\mathbb{K} \cup\{\infty\}$. So, there are three values $t_{1}, t_{2}, t_{3} \in \mathbb{K} \cup\{\infty\}$ such that $v\left(t_{i}\right)=Y_{i}$. Let $\tau(t) \in \mathbb{K}(t)$ be the unique unit associated to the transformation of the projective line $\mathbb{P}(\mathbb{F})$ into itself given by $\tau(0)=t_{1}, \tau(1)=t_{2}, \tau(\infty)=t_{3}$. Then $v(\tau(t))=u(t)$ and both units represent the same hypercircle.

\section{Parametrization And implicitation of A hyPercircle}

In this section, we will provide specific methods to parametrize and implicitate hypercircles. These methods show the power of the rich structure of hypercircles, simplifying problems that are usually much harder in general.

Given a unit $u(t)$ defining $\mathcal{U}$, it is immediate to obtain a parametrization of $\mathcal{U}$ (see Section 2). If $\mathcal{U}$ is given by implicit equations (as is usually the case in Weil's descente method), the next proposition shows how to parametrize it.

Proposition 5.1. The pencil of hyperplanes $X_{0}+X_{1} \alpha+\cdots+X_{n-1} \alpha^{n-1}=t$ parametrizes the primitive $\alpha$-hypercircle $\mathcal{U}$.

Proof. Let $I$ be the implicit ideal of $\mathcal{U}$. Note that, since $\mathcal{U}$ is $\mathbb{K}$-rational it is $\mathbb{K}$ definable, and hence a set of generators of $I$ can be taken in $\mathbb{K}\left[X_{0}, \ldots, X_{n-1}\right]$. Let $u(t)$ be any unit associated with $\mathcal{U}$ and $\left(\phi_{0}(t), \ldots, \phi_{n-1}(t)\right)$ the induced parametrization. Let $v(t)$ be the inverse unit of $u(t), u(v(t))=v(u(t))=t$. Then $\left(\phi_{0}(v(t)), \ldots\right.$, $\left.\phi_{n-1}(v(t))\right)=\left(\psi_{0}(t), \ldots, \psi_{n-1}(t)\right)=\Psi(t)$ is another parametrization of $\mathcal{U}$ which is no longer defined over $\mathbb{K}$ but over $\mathbb{K}(\alpha)$. The later parametrization is in standard form [12, that is,

$$
\sum_{i=0}^{n-1} \psi_{i}(t) \alpha^{i}=\left(\sum_{i=0}^{n-1} \phi_{i}(t) \alpha^{i}\right) \circ v(t)=u \circ v(t)=t .
$$

This implies that the pencil of hyperplanes $H_{t} \equiv X_{0}+X_{1} \alpha+\cdots+X_{n-1} \alpha^{n-1}-t$ parametrizes $\mathcal{U}$. Indeed, if $\Psi(t)$ is defined, $H_{t} \cap \mathcal{U}$ consists in $n-1$ points at infinity of $\mathcal{U}$ (Theorem 4.2) and $\Psi(t)$ itself. We deduce that $\psi_{i}(t)-X_{i}$ belongs to the ideal $I+H_{t}$, which has a set of generators in $\mathbb{K}(\alpha)(t)\left[X_{0}, \ldots, X_{n-1}\right]$. So, the parametrization $\Psi(t)$ can be computed from $I$.

Notice that the obtained parametrization $\Psi(t)$ has coefficients over $\mathbb{K}(\alpha)$. Thus, it is not the parametrization induced by any associated unit $u(t)$. The interest of obtaining a unit associated to a hypercircle is that it helps us to solve the problem of reparametrizing a curve over an optimal field extension of $\mathbb{K}$; see [4]. There, it is shown that given a parametrization $\Psi(t) \in \mathbb{K}(\alpha)^{r}$ of a curve there is a hypercircle associated to it. Any unit associated to the hypercircle reparametrizes the original curve over $\mathbb{K}$. To get a parametrization $\phi(t)$ over $\mathbb{K}$ or, equivalently, a unit $u(t)$ associated to $\mathcal{U}$, we refer to 12 . In addition, note that the proof of Proposition 4.7 shows how to construct a unit associated to a hypercircle, when points over $\mathbb{K}$ are known, and therefore a parametrization of it.

The inverse problem, computing implicit equations of a hypercircle from the parametrization induced by an associated unit, can be performed using classic implicitation methods. However, the special structure of hypercircles provides specific methods that might be more convenient. 
Proposition 5.2. Let $\mathcal{U}$ be a hypercircle associated to the unit $u(t)$, and let $v(t)$ be the inverse of $u(t)$. Let

$$
v\left(\sum_{i=0}^{n-1} \alpha^{i} X_{i}\right)=\sum_{i=0}^{n-1} \frac{r_{i}\left(X_{0}, \ldots, X_{n-1}\right)}{s\left(X_{0}, \ldots, X_{n-1}\right)} \alpha^{i},
$$

where $r_{i}, s \in \mathbb{K}\left[X_{0}, \ldots, X_{n-1}\right]$. Then, the ideal of $\mathcal{U}$ is the elimination ideal with respect to $Z$ :

$$
\mathcal{I}(\mathcal{U})=\left(r_{1}(\bar{X}), \ldots, r_{n}(\bar{X}), s(\bar{X}) Z-1\right) \cap \mathbb{F}\left[X_{0}, \ldots, X_{n-1}\right] .
$$

Proof. Let $u(t)=\frac{a t+b}{t+d}$, then $v(t)=\frac{-d t+b}{t-a}$. Now, consider

$$
\begin{aligned}
u\left(\sum_{i=0}^{n-1} \alpha^{i} X_{i}\right) & =\sum_{i=0}^{n-1} \xi_{i}\left(X_{0}, \ldots, X_{n-1}\right) \alpha^{i}, \\
v\left(\sum_{i=0}^{n-1} \alpha^{i} X_{i}\right) & =\sum_{i=0}^{n-1} \eta_{i}\left(X_{0}, \ldots, X_{n-1}\right) \alpha^{i},
\end{aligned}
$$

where $\xi_{i}, \eta_{j} \in \mathbb{K}\left(X_{0}, \ldots, X_{n-1}\right)$ and $\eta_{i}=\frac{r_{i}\left(X_{0}, \ldots, X_{n-1}\right)}{s\left(X_{0}, \ldots, X_{n-1}\right)}$. The map $\xi: \mathbb{F}^{n} \longrightarrow \mathbb{F}^{n}$, $\xi=\left(\xi_{0}, \ldots, \xi_{n-1}\right)$ is birational and its inverse is $\eta=\left(\eta_{0}, \ldots, \eta_{n-1}\right)$. Indeed,

$$
\begin{aligned}
\sum_{i=0}^{n-1} \eta_{i}\left(\xi_{0}(\bar{X}), \ldots, \xi_{n-1}(\bar{X})\right) \alpha^{i} & =v\left(\sum_{j=0}^{n-1} \alpha^{j} \xi_{j}(\bar{X})\right) \\
=v\left(u\left(\sum_{i=0}^{n-1} \alpha^{i} X_{i}\right)\right) & =\sum_{i=0}^{n-1} \alpha^{i} X_{i}
\end{aligned}
$$

is an equality in $\mathbb{K}(\alpha)\left(X_{0}, \ldots, X_{n-1}\right)$. We deduce that

$$
\eta_{i}\left(\xi_{0}\left(X_{0}, \ldots, X_{n-1}\right), \ldots, \xi_{n-1}\left(X_{0}, \ldots, X_{n-1}\right)\right)=X_{i} .
$$

It is clear that $\mathcal{U}$ is the image of the line $L \equiv\left\{X_{1}=0, \ldots, X_{n-1}=0\right\}$ under the map $\xi, \mathcal{U}=\xi(L)$. The set of points where $\xi$ is not defined is the union of the hyperplanes $\sum_{i=0}^{n-1} \sigma_{j}(\alpha)^{i} X_{i}+\sigma_{j}(d)=0,1 \leq j \leq n$. The intersection of these hyperplanes with $L$ is the set of points $\left(-\sigma(d)_{j}, 0, \ldots, 0\right), 1 \leq j \leq n$. Thus, for a generic $p \in L, \xi(p)$ is defined and belongs to $\mathcal{U}$. The result is similar for the inverse map $\eta$. The set of points where $\eta$ is not defined is the union of the hyperplanes $\sum_{i=0}^{n-1} \sigma_{j}(\alpha)^{i} X_{i}-\sigma_{j}(a)=0,1 \leq j \leq n$. These $n$ hyperplanes intersect $\mathcal{U}$ in at most one affine point; see Proposition [5.1. So, for a generic $p \in \mathcal{U}, \eta(p)$ is again defined and belongs to $L$. Let us now compute the points $\bar{X}$ such that $\eta(\bar{X})$ is defined, but it does not belong to the domain of $\xi$. If $\bar{X}$ is such a point, then

$$
\sum_{i=0}^{n-1} \sigma_{j}(\alpha)^{i} \eta_{i}(\bar{X})+\sigma_{j}(d)=0 .
$$

As $\eta_{i}$ is defined over $\mathbb{K}$, applying $\sigma_{j}$ to the definition of $\eta$, we obtain that

$$
\sigma_{j}(v)\left(\sum_{i=0}^{n-1} \sigma_{j}(\alpha)^{i} X_{i}\right)=-\sigma_{j}(d)
$$

But $\sigma_{j}(v)=\frac{-\sigma_{j}(d) t+\sigma_{j}(b)}{t-\sigma_{j}(a)}$. It follows from Lemma 4.6 that the value $-\sigma_{j}(d)$ cannot be reached, even in $\mathbb{F}$. Thus, the image of $\eta$ is contained in the domain of $\xi$. 
We are ready to prove the theorem, by verifying that the set $\mathcal{U} \backslash\{s=0\}$, which is just eliminating a finite number of points in $\mathcal{U}$, is the set of points $\bar{X}$ such that $r_{i}(\bar{X})=0, i \geq 1$ and $s(\bar{X}) \neq 0$. If $\bar{X} \in \mathcal{U} \backslash\{s=0\}$, then $\eta$ is defined and $\eta(\bar{X})=\left(\eta_{0}(\bar{X}), 0, \ldots, 0\right)$. Hence $\eta_{i}(\bar{X})=r_{i}(\bar{X})=0$. Conversely, if $\bar{X}$ is a point such that $r_{i}(\bar{X})=0$ and $s(\bar{X}) \neq 0$, then $\eta(\bar{X})$ is defined and belongs to $L$. It is proven that $\xi$ is defined in $\eta(\bar{X})$, so $\bar{X}=\xi(\eta(\bar{X})) \in \xi(L)=\mathcal{U}$. The thesis of the theorem follows taking the Zariski closure of $\mathcal{U} \backslash\{s=0\}$.

This method to compute the implicit equations of $\mathcal{U}$ is not free from elimination techniques, as it has to eliminate the variable $Z$. However, it has the advantage that it yields already an ideal in $\mathbb{F}\left[X_{0}, \ldots, X_{n-1}\right]$ defined over $\mathbb{K}$ and such that it describes a nontrivial variety containing the hypercircle. Namely, $\left(r_{1}(\bar{X}), \ldots, r_{n-1}(\bar{X})\right)$ are polynomials over $\mathbb{K}$ whose zero set contains the hypercircle. The following example shows that the elimination step is necessary in some cases.

Example 5.3. Let $\mathbb{Q} \subseteq \mathbb{Q}(\alpha)$ be the algebraic extension defined by $\alpha^{3}+\alpha^{2}-3=$ 0 . Let us consider the unit $u(t)=\frac{(2+\alpha) t+\alpha}{t+1-\alpha}$. Its inverse is $v(t)=\frac{(\alpha-1) t+\alpha}{t-2-\alpha}$. A parametrization of $\mathcal{U}$ is

$$
\phi(t)=\left(\frac{2 t^{3}+6 t^{2}+7 t+3}{t^{3}+4 t^{2}+5 t-1}, \frac{t^{3}+6 t^{2}+9 t+2}{t^{3}+4 t^{2}+5 t-1}, \frac{t^{2}+4 t+1}{t^{3}+4 t^{2}+5 t-1}\right) .
$$

A Gröbner basis of the ideal of the curve is

$$
\begin{aligned}
I:=\left\{x_{1}^{2}-x_{2} x_{0}-x_{2} x_{1}-x_{1}+x_{2}, x_{0} x_{1}-\right. & x_{2} x_{0}-3 x_{2}^{2}-2 x_{1}+4 x_{2}, \\
& \left.x_{0}^{2}-3 x_{2} x_{1}-2 x_{0}+2 x_{1}+3 x_{2}-2\right\} .
\end{aligned}
$$

Then, Proposition 5.2 states that this ideal is

$$
I=\left(r_{1}\left(x_{0}, x_{1}, x_{2}\right), r_{2}\left(x_{0}, x_{1}, x_{2}\right), s\left(x_{0}, x_{1}, x_{2}\right) Z-1\right) \cap \mathbb{F}\left[x_{0}, x_{1}, x_{2}\right]
$$

where

$$
\begin{aligned}
r_{1}= & 2-8 x_{2}+4 x_{2} x_{0}+6 x_{2}^{2} x_{0}+17 x_{2} x_{1}+x_{2} x_{0}^{2}+3 x_{1}-3 x_{1}^{2} x_{2}+x_{0}^{3}-x_{0}^{2} x_{1} \\
& +4 x_{0} x_{1}-12 x_{2}^{2}-8 x_{1}^{2}+9 x_{2}^{3}+3 x_{1}^{3}-3 x_{0}^{2}-9 x_{0} x_{1} x_{2}, \\
r_{2}= & -2-7 x_{2}+4 x_{2} x_{0}-x_{2} x_{1}+8 x_{1}-2 x_{0}-2 x_{0} x_{1}+6 x_{2}^{2}-2 x_{1}^{2}+x_{0}^{2}, \\
s= & 9 x_{2}^{3}+6 x_{2}^{2} x_{0}-12 x_{2}^{2}+5 x_{2} x_{0}-17 x_{2}-3 x_{1}^{2} x_{2}-9 x_{0} x_{1} x_{2}+x_{2} x_{0}^{2}+24 x_{2} x_{1} \\
& +3 x_{1}^{3}+8 x_{0}+4 x_{0} x_{1}-5 x_{0}^{2}-x_{0}^{2} x_{1}+5 x_{1}-9 x_{1}^{2}-7+x_{0}^{3} .
\end{aligned}
$$

But, if we take $J=\left(r_{1}, r_{2}\right)$, then $J \subsetneq I$. The saturation of $J$ with respect to $I$ is

$$
\begin{aligned}
J: I^{\infty}=\left(x_{1}^{2}-x_{0} x_{2}-x_{1} x_{2}-2 x_{1}+\right. & 3 x_{2}+1, x_{0} x_{1}-x_{0} x_{2}-3 x_{2}^{2}-x_{0}-2 x_{1} \\
& \left.+2 x_{2}+2, x_{0}^{2}-3 x_{1} x_{2}-4 x_{0}+3 x_{2}+4\right) .
\end{aligned}
$$

This ideal corresponds to the union of the line

$$
\left\{\begin{aligned}
-\alpha x_{0}+3 x_{2} & =-2 \alpha \\
\left(\alpha+\alpha^{2}\right) x_{0}-3 x_{1} & =-3+2 \alpha+2 \alpha^{2}
\end{aligned}\right.
$$

and its conjugates. 
The next theorem shows an alternative method to implicitate a hypercircle without using any elimination techniques. It is based on properties of the normal rational curve of degree $n$.

Theorem 5.4. Let $\varphi(t)=\left(\frac{q_{0}(t)}{N(t)}, \ldots, \frac{q_{n-1}(t)}{N(t)}\right)$ be a proper parametrization of a primitive hypercircle $\mathcal{U}$ with coefficients in $\mathbb{F}$. Let $I$ be the homogeneous ideal of the rational normal curve of degree $n$ in $\mathbb{P}(\mathbb{F})^{n}$ given by a set of homogeneous generators $h_{1}(\bar{Y}), \ldots, h_{r}(\bar{Y})$. Let $\mathcal{Q} \in \mathcal{M}_{n+1 \times n+1}(\mathbb{F})$ be the matrix that carries $\left\{q_{0}(t), \ldots, q_{n-1}(t), N(t)\right\}$ onto $\left\{1, t, \ldots, t^{n}\right\}$. Let

$$
f_{i}(\bar{X})=h_{i}\left(\sum_{j=0}^{n} \mathcal{Q}_{0 j} X_{j}, \ldots, \sum_{j=0}^{n} \mathcal{Q}_{n j} X_{j}\right), 1 \leq i \leq r .
$$

Then $\left\{f_{1}, \ldots, f_{r}\right\}$ is a set of generators of the homogeneous ideal of $\mathcal{U}$.

Proof. If the parametrization is proper, then $\left\{q_{0}(t), \ldots, q_{n-1}(t), N(t)\right\}$ is a basis of the polynomials of degree at most $n$. This follows from the fact shown in Corollary 3.2 that a primitive hypercircle is not contained in any hyperplane. Note that a projective point $\bar{X}$ belongs to $\mathcal{U}$ if and only if $\mathcal{Q}(\bar{X})$ belongs to the rational normal curve, if and only if $h_{i}(\mathcal{Q}(\bar{X}))=0,1 \leq i \leq r$.

\section{Remark 5.5.}

- It is well known that the set of polynomials $\left\{Y_{i} Y_{j-1}-Y_{i-1} Y_{j} \mid 1 \leq i, j \leq n\right\}$ is a generator set of $I$ (see [6]).

- Notice that it is straightforward to compute $Q$ from the parametrization. Therefore, we have an effective method to compute the implicit ideal of the projective closure of $\mathcal{U}$. The affine ideal of $\mathcal{U}$ can be obtained by dehomogenization $X_{n}=1$.

- If the parametrization is given by polynomials over an algebraic extension $\mathbb{K}(\beta)$ of $\mathbb{K}$, then the coefficients of $f_{i}$ belongs to $\mathbb{K}(\beta)$. Moreover, if we write $f_{i}(\bar{X})=\sum_{j=0}^{m} f_{i j}(\bar{X}) \beta^{j}$, with $f_{i j} \in \mathbb{K}[\bar{X}]$, then, $\left\{f_{i j}\right\}$ is a set of generators over $\mathbb{K}$ of the hypercircle $\mathcal{U}$.

- In practice, this method is much more suited to compute an implicitation of a hypercircle than the method presented in Proposition 5.2 .

Example 5.6. The implicit equations of a hypercircle can be computed by classical implicitation methods, for example, the Gröbner basis method or with the two methods presented in Proposition 5.2 and Theorem 5.4. Here, we present two cases that show the practical behavior of these methods. The first example considers the algebraic extension $\mathbb{Q} \subseteq \mathbb{Q}(\alpha)$, where $\alpha^{4}+\alpha^{2}-3$ and the unit $u=\frac{\left(1-\alpha^{3}\right) t+\alpha^{2}}{t+1+2 \alpha-3 \alpha^{2}}$. The parametrization of the hypercircle is given by

$$
\begin{aligned}
& \phi_{0}=\frac{t^{4}+15 t^{3}+22 t^{2}+101 t-195}{t^{4}+10 t^{3}-17 t^{2}-366 t+233}, \phi_{1}=\frac{-11 t^{3}-73 t^{2}+65 t-114}{t^{4}+10 t^{3}-17 t^{2}-366 t+233}, \\
& \phi_{2}=\frac{2 t^{3}+57 t^{2}-25 t-59}{t^{4}+10 t^{3}-17 t^{2}-366 t+233}, \phi_{3}=\frac{-t^{4}-6 t^{3}+4 t^{2}+17 t-56}{t^{4}+10 t^{3}-17 t^{2}-366 t+233} .
\end{aligned}
$$

The second example starts from the extension $\mathbb{Q} \subseteq \mathbb{Q}(\beta)$, where $\beta$ is such that $\beta^{4}+$ $3 \beta+1=0$. Here, the unit defining $\mathcal{U}$ is $u=\frac{\left(1+\beta-\beta^{2}\right) t+1+\beta^{3}}{t+1+\beta^{2}-\beta^{3}}$ and the parametrization 
induced by $u(t)$ is

$$
\begin{aligned}
& \psi_{0}=\frac{t^{4}+11 t^{3}+47 t^{2}+95 t+72}{t^{4}+13 t^{3}+62 t^{2}+126 t+81}, \psi_{1}=\frac{t^{4}+7 t^{3}+15 t^{2}+17 t+9}{t^{4}+13 t^{3}+62 t^{2}+126 t+81}, \\
& \psi_{2}=\frac{-t^{4}-10 t^{3}-31 t^{2}-23 t}{t^{4}+13 t^{3}+62 t^{2}+126 t+81}, \psi_{3}=\frac{t^{3}+13 t^{2}+42 t+36}{t^{4}+13 t^{3}+62 t^{2}+126 t+81} .
\end{aligned}
$$

The running times for computing the implicit ideal (using a Mac Xserver with 2 processors G5 $2.3 \mathrm{GHz}, 2$ Gb RAM Maple 10) are

\begin{tabular}{|l|l|l|}
\hline & Example 1 & Example 2 \\
\hline Gröbner basis method & 0.411 & 0.332 \\
Proposition 5.2 & 2.094 & 2.142 \\
Theorem 5.4 & 0.059 & 0.021 \\
\hline
\end{tabular}

We refer the interested reader to [11] for a brief discussion and comparison of the running times of these algorithms.

\section{Characterization of hypercircles}

In the introduction, we defined algebraically a circle as the conic such that its homogeneous part is $x^{2}+y^{2}$ and contains an infinite number of real points. The condition on the homogeneous part is equivalent to impose that the curve passes through the points at infinity $[ \pm i: 1: 0]$. Analogously, hypercircles are regular curves of degree $n$ with infinite points over the base field passing through the points at infinity described in Theorem 4.2. The following result shows that this is a characterization of these curves.

Theorem 6.1. Let $\mathcal{U} \subseteq \mathbb{F}^{n}$ be an algebraic set of degree $n$ such that all those components are of dimension 1. Then, it is a primitive $\alpha$-hypercircle if and only if it has an infinite number of points with coordinates in $\mathbb{K}$ and passes through the set of points at infinity characterized in Theorem 4.2 .

Proof. The only if implication is trivial. For the other one, let $\mathcal{U} \subseteq \mathbb{F}^{n}$ be an algebraic set of pure dimension 1 and degree $n$ passing through $P=\left\{P_{1}, \ldots, P_{n}\right\}$, which are the $n$ points at infinity of a primitive $\alpha$-hypercircle. Suppose that $\mathcal{U}$ has infinite points with coordinates in $\mathbb{K}$. Then, we are going to prove that $\mathcal{U}$ is irreducible. Let $\mathcal{W}$ be an irreducible component of $\mathcal{U}$ with infinite points in $\mathbb{K}$. Note that, since $\mathcal{W}$ is irreducible and contains infinitely many points over $\mathbb{K}$, the ideal $\mathcal{I}(\mathcal{W})$ over $\mathbb{F}$ is generated by polynomials over $\mathbb{K}$ (see Lemma 2 in $[3]$ ). Let $q$ be any point at infinity of $\mathcal{W}$; then $q \in P$. As $\mathcal{W}$ is $\mathbb{K}$-definable, it follows that $\mathcal{W}$ also contains all conjugates of $q$. Thus, $P$ is contained in the set of points at infinity of $\mathcal{W}$. It follows that $\mathcal{W}$ is of degree at least $n$; since $\mathcal{W} \subseteq \mathcal{U}, \mathcal{U}=\mathcal{W}$. Therefore, $\mathcal{U}$ is irreducible and $\mathcal{I}(\mathcal{U})$ is generated by polynomials with coefficients over $\mathbb{K}$. Now, consider the pencil of hyperplanes $H_{t} \equiv X_{0}+X_{1} \alpha+\cdots+X_{n-1} \alpha^{n-1}-t$, where $t$ takes values in $\mathbb{F}$. Notice that $\overline{H_{t}} \cap P=\left\{P_{2}, \ldots, P_{n}\right\}$. Thus, $P_{1} \in \overline{\mathcal{U}} \backslash \overline{H_{t}}$ so, for all $t, \mathcal{U} \nsubseteq H_{t}$. Moreover, for every point $p=\left(p_{0}, \ldots, p_{n-1}\right) \in \mathcal{U}, t(p)=\sum_{i=0}^{n-1} p_{i} \alpha^{i} \in \mathbb{F}$ is such that $\bar{H}_{t(p)} \cap \overline{\mathcal{U}}=\left\{p, P_{2}, \ldots, P_{n}\right\}$. The cardinal of $\{t(p) \mid p \in \mathcal{U}\}$ is infinite, since otherwise, by the irreducibility of $\mathcal{U}$, it would imply that there is a $t_{0}$ such that $\mathcal{U} \subseteq H_{t_{0}}$, which is impossible. So, for generic $t$, the intersection is $\bar{H}_{t} \cap \overline{\mathcal{U}}=$ $\left\{p(t), P_{2}, \ldots, P_{n}\right\}$. Let us check that the coordinates of $p(t)$ are rational functions in $\mathbb{K}(\alpha)(t)$. Take the ideal $\mathcal{I}(\mathcal{U})$ of $\mathcal{U}$. The ideal of $p(t)$ (as a point in $\left.\mathbb{F}(t)^{n}\right)$ is $I+H_{t}$, 
defined over $\mathbb{K}(\alpha)(t)$. The reduced Gröbner basis of the radical $I+H_{t}$ is of this kind $\left(X_{0}-\psi_{0}, \ldots, X_{n-1}-\psi_{n-1}\right)$ and it is also defined over $\mathbb{K}(\alpha)(t)\left[X_{0}, \ldots, X_{n-1}\right]$. Hence, $\left(\psi_{0}, \ldots, \psi_{n-1}\right)$ is a $\mathbb{K}(\alpha)$-parametrization of $\mathcal{U}$. Thus, since $\mathcal{U}$ is irreducible, it is rational. Moreover $\sum_{i=0}^{n-1}\left(\psi_{i}(t)\right) \alpha^{i}=t$ and the parametrization is proper. As the curve is rational and has an infinite number of points over $\mathbb{K}$, it is parametrizable over $\mathbb{K}$ (it follows, for example, from the results in [15]). Let $u(t)$ be a unit such that $\Psi \circ u(t)=\left(\phi_{0}(t), \ldots, \phi_{n-1}(t)\right)$ is a parametrization over $\mathbb{K}$, where $\phi_{i}(t) \in \mathbb{K}(t)$ and $\sum_{i=0}^{n-1} \phi_{i}(t) \alpha^{i}=u(t)$. We conclude that $\mathcal{U}$ is the hypercircle associated to the unit $u(t)$.

Remark that a parametric curve, definable over $\mathbb{K}$ and with a regular point over $\mathbb{K}$, is parametrizable over the same field; for this, it is enough to $\mathbb{K}$-birationally project the curve over a plane, such that the $\mathbb{K}$-regular point stays regular on the projection, and then apply the results in [15. Then, a small modification of the proof above, yields the following:

Theorem 6.2. Let $\mathcal{U} \subseteq \mathbb{F}^{n}$ be a 1-dimensional irreducible algebraic set of degree $n$, definable over $\mathbb{K}$. Then, it is a primitive $\alpha$-hypercircle if and only if it has a regular point with coordinates in $\mathbb{K}$ and passes through the set of points at infinity characterized in Theorem 4.2

\section{An APPLICATION}

As mentioned in the introduction, hypercircles play an important role in the problem of the optimal-algebraic reparametrization of a rational curve (see [3], 4], 12 13, 14 17 for further details). Roughly speaking, the problem is as follows. Given a rational $\mathbb{K}$-definable curve $\mathcal{C}$ by means of a proper rational parametrization over $\mathbb{K}(\alpha)$, decide whether $\mathcal{C}$ can be parametrized over $\mathbb{K}$ and, in the affirmative case, find a change of parameter transforming the original parametrization into a parametrization over $\mathbb{K}$. In [4], a $\mathbb{K}$-definable algebraic variety in $\mathbb{F}^{n}$, where $n=[\mathbb{K}(\alpha): \mathbb{K}]$, is associated to $\mathcal{C}$. This variety is called the associated Weil (descent) parametric variety. In [4, it is proved that this Weil variety has exactly one one-dimensional component iff $\mathcal{C}$ is $\mathbb{K}$-definable (which is our case) and, in this case, $\mathcal{C}$ can be parametrized over $\mathbb{K}$ iff this one-dimensional component is a $\mathbb{K}, \alpha$ hypercircle 1 . Moreover, if it is a hypercircle a proper rational parametrization over $\mathbb{K}$ of the hypercircle generates the change of parameter one is looking for; namely its generating unit.

In the following example, we illustrate how to use the knowledge of the geometry of hypercircles to help solve the problem. Suppose given the parametric curve

$$
\begin{gathered}
\mathcal{C} \simeq\left(\eta_{1}(t), \eta_{2}(t)\right) \\
=\left(\frac{\left(-2 t^{4}-2 t^{3}\right) \alpha-2 t^{4}}{6 \alpha^{2} t^{2}+\left(4 t^{3}-2\right) \alpha+t^{4}-8 t}, \frac{-2 t^{4} \alpha}{6 \alpha^{2} t^{2}+\left(4 t^{3}-2\right) \alpha+t^{4}-8 t}\right)
\end{gathered}
$$

where $\alpha$ is algebraic over $\mathbb{Q}$ with minimal polynomial $x^{3}+2$. We follow Weil's descente method presented in [4] to associate a hypercircle to $\mathcal{C}$. The method

\footnotetext{
${ }^{1}$ As remarked by a referee, see also [17, this component will always be an $\alpha$-hypercircle over some other base field.
} 
consists of writing $\eta_{i}\left(\sum_{j=0}^{2} t_{j} \alpha^{j}\right)=\sum_{j=0}^{2} \frac{q_{i j}\left(t_{0}, t_{1}, t_{2}\right)}{N\left(t_{0}, t_{1}, t_{2}\right)}$. In this situation $\mathcal{C}$ is $\mathbb{Q}$ definable if and only if

$$
\mathcal{V}=\overline{V\left(q_{11}, q_{12}, q_{21}, q_{22}\right) \backslash V(N)}
$$

is of dimension 1. Moreover, $\mathcal{C}$ is $\mathbb{Q}$-parametrizable if and only if the one-dimensional component of $\mathcal{V}$ is an $\alpha$-hypercircle. For this example, the equations of $\mathcal{V}$ are:

$\mathcal{V}=V\left(2 t_{0}^{3} t_{2}-4 t_{2}^{4}+3 t_{0}^{2} t_{1}^{2}+2 t_{1}^{3} t_{2}+2 t_{0} t_{2}^{2}+2 t_{1}^{2} t_{2}-t_{0}^{2} t_{1}+6 t_{0} t_{1} t_{2}^{2},-6 t_{0}^{2} t_{1} t_{2}+t_{0}^{4}+2 t_{0} t_{1}^{2}-\right.$ $8 t_{0} t_{2}^{3}-2 t_{0} t_{1}^{3}+2 t_{0}^{2} t_{2}-4 t_{1} t_{2}^{2}-12 t_{1}^{2} t_{2}^{2}, 12 t_{2}^{2} t_{1}^{3}-9 t_{0} t_{1} t_{2}^{3}+6 t_{2}^{5}-4 t_{0} t_{1}^{3}-2 t_{0}^{2} t_{1} t_{2}+4 t_{1}^{2} t_{2}^{2}-$ $4 t_{0} t_{2}^{3}, 9 t_{0} t_{1}^{2} t_{2}^{2}-9 t_{0}^{2} t_{2}^{3}-2 t_{0}^{3} t_{2}-2 t_{1}^{3} t_{2}+6 t_{0} t_{1} t_{2}^{2}-2 t_{2}^{4}+t_{0}^{2} t_{1}-2 t_{1}^{2} t_{2}-2 t_{0} t_{2}^{2}, 6 t_{0}^{2} t_{1} t_{2}^{2}+12 t_{1}^{2} t_{2}^{3}-$ $t_{0}^{3} t_{1}-2 t_{0} t_{1}^{2} t_{2}-2 t_{0}^{2} t_{2}^{2}+8 t_{1} t_{2}^{3}, 6 t_{0}^{3} t_{2}^{2}+9 t_{0} t_{1} t_{2}^{3}-6 t_{2}^{5}+2 t_{0} t_{1}^{3}-2 t_{0}^{2} t_{1} t_{2}+4 t_{1}^{2} t_{2}^{2}+8 t_{0} t_{2}^{3}, 18 t_{2} t_{1}^{4}+$ $36 t_{2}^{4} t_{1}+14 t_{0}^{3} t_{2}+32 t_{1}^{3} t_{2}+12 t_{0} t_{1} t_{2}^{2}-4 t_{2}^{4}-7 t_{0}^{2} t_{1}+14 t_{1}^{2} t_{2}+14 t_{0} t_{2}^{2}, 6 t_{0} t_{1}^{3} t_{2}+2 t_{0} t_{1}^{2} t_{2}+$ $t_{0}^{3} t_{1}+2 t_{0}^{2} t_{2}^{2}-8 t_{1} t_{2}^{3}+12 t_{2}^{4} t_{0}, 9 t_{0}^{3} t_{2} t_{1}-36 t_{2}^{4} t_{1}-4 t_{0}^{3} t_{2}-4 t_{1}^{3} t_{2}+12 t_{0} t_{1} t_{2}^{2}-4 t_{2}^{4}+2 t_{0}^{2} t_{1}-$ $4 t_{1}^{2} t_{2}-4 t_{0} t_{2}^{2}, 6 t_{1}^{5}+48 t_{1}^{2} t_{2}^{3}-36 t_{2}^{4} t_{0}-11 t_{0}^{3} t_{1}+6 t_{1}^{4}+14 t_{0} t_{1}^{2} t_{2}-22 t_{0}^{2} t_{2}^{2}+64 t_{1} t_{2}^{3}, 3 t_{1}^{4} t_{0}+$ $6 t_{0} t_{1} t_{2}^{3}+2 t_{0} t_{1}^{3}+t_{0}^{2} t_{1} t_{2}-2 t_{1}^{2} t_{2}^{2}+2 t_{0} t_{2}^{3}, 27 t_{2}^{4} t_{1}^{2}-27 t_{0} t_{2}^{5}-9 t_{0}^{2} t_{2}^{3}+9 t_{2}^{4} t_{1}-2 t_{0}^{3} t_{2}-2 t_{1}^{3} t_{2}+$ $\left.6 t_{0} t_{1} t_{2}^{2}-2 t_{2}^{4}+t_{0}^{2} t_{1}-2 t_{1}^{2} t_{2}-2 t_{0} t_{2}^{2}, 6 t_{2}^{4} t_{0}^{2}+12 t_{2}^{5} t_{1}-5 t_{0} t_{1} t_{2}^{3}+2 t_{2}^{5}, t_{0} t_{2}^{5} t_{1}+2 t_{2}^{7}\right)$.

Thus the main point is to verify that this curve is a $\mathbb{K}, \alpha$-hypercircle. Now, following Proposition 5.1, we may try to parametrize $\mathcal{V}$ by the pencil of hyperplanes $t_{0}+\alpha t_{1}+\alpha^{2} t_{2}-t$. Doing so, we obtain the parametrization

$$
\left(\frac{\left(\alpha^{2}+2 \alpha t+t^{2}\right) t}{3 \alpha t+\alpha^{2}+3 t^{2}}, \frac{-1 / 2 \alpha^{2} t^{3}}{3 \alpha t+\alpha^{2}+3 t^{2}}, \frac{-1 / 2 \alpha t^{2}(t+\alpha)}{3 \alpha t+\alpha^{2}+3 t^{2}}\right) .
$$

Remark that this parametrization can also be computed by means of inverse computation techniques as described in 14. Then, by direct computation, we observe that the parametric irreducible curve defined by this parametrization is of degree 3 , passes through the point $(0,0,0)$ and this point is regular. Moreover, it is $\mathbb{Q}$ definable, since it is the only 1-dimensional component of $\mathcal{V}$ (see [4), which is, by construction, a $\mathbb{Q}$-definable variety. It follows from 6.2 that it is a $\mathbb{K}, \alpha$-hypercircle.

Then, from this parametrization, the algorithm presented in [12] computes a unit $u(t)=\frac{2}{2 t+\alpha^{2}}$ associated to $\mathcal{V}$. So, $\mathcal{V}$ is the hypercircle associated to $u(t)$ and $\mathcal{C}$ is parametrizable over $\mathbb{Q}$. In particular, the parametrization of $\mathcal{V}$ associated to $u(t)$ is $\left(\frac{2 t^{2}}{2 t^{3}+1}, \frac{-1}{2 t^{3}+1}, \frac{-t}{2 t^{3}+1}\right)$. Moreover, the unit $u(t)$ gives the change of parameter we need to compute a parametrization of $\mathcal{C}$ over the base field (see [4]), namely:

$$
\eta(u(t))=\left(\frac{t+1}{t^{4}}, \frac{1}{t^{4}}\right) .
$$

It is plausible to consider there are some advantages on performing the reduction, from the given simplification problem of the parametrization over $\mathbb{K}(\alpha)$ of $\mathcal{C}$ to searching for a $\mathbb{K}$-parametrization of $\mathcal{V}$. In fact:

- Implicit equations for $\mathcal{V}$, and a parametrization of this variety over $\mathbb{K}(\alpha)$, are provided by construction (respectively, by Proposition 5.1).

- The degree of $\mathcal{V}$ is always the degree of the algebraic extension (Corollary 3.2). So, the complexity of the geometric object $\mathcal{V}$ could be considerably smaller than that of $\mathcal{C}$ (depending on the degree of the latter).

- Thus, searching for $\mathbb{K}$-rational points over $\mathcal{V}$ seems simpler than for $\mathcal{C}$ because we have a lot of information on the geometry of $\mathcal{V}$ and, also, because its degree could be much smaller than that of $\mathcal{C}$. 
The well-known theorem of Hilbert and Hurwitz ([8, [16]) is often used in the reparametrization problem, as it reduces, after performing a series of birational transformations, the finding of rational points on $\mathcal{C}$ to the case of conics or lines. What we propose here could be regarded as a variant of that procedure, in which the final search has to be done with hypercircles, curves of higher degree than conics, but obtained in a much simpler way, and sharing many properties with conics.

In particular, we think that future work should be devoted to continue the study of the properties of hypercircles, focusing on finding $\mathbb{K}$-rational points.

\section{REFERENCES}

[1] Cesar Alonso, Jaime Gutiérrez, and Tomás Recio, A rational function decomposition algorithm by near-separated polynomials, J. Symbolic Comput. 19 (1995), no. 6, 527-544. MR:1370620 (96j:13025)

[2] Carlos Andradas and Tomás Recio, Plotting missing points and branches of real parametric curves, Appl. Algebra Engrg. Comm. Comput. 18 (2007), no. 1-2, 107-126. MR.2280313

[3] Carlos Andradas, Tomás Recio, and J. Rafael Sendra, A relatively optimal rational space curve reparametrization algorithm through canonical divisors, Proceedings of the 1997 International Symposium on Symbolic and Algebraic Computation (Kihei, HI) (New York), ACM, 1997, pp. 349-355 (electronic). MR 1810004

[4] - Base field restriction techniques for parametric curves, Proceedings of the 1999 International Symposium on Symbolic and Algebraic Computation (Vancouver, BC) (New York), ACM, 1999, pp. 17-22 (electronic). MR1802062 (2002b:14080)

[5] David Cox, John Little, and Donal O'Shea, Ideals, varieties, and algorithms, second ed., Undergraduate Texts in Mathematics, Springer-Verlag, New York, 1997, An introduction to computational algebraic geometry and commutative algebra. MR:1417938 (97h:13024)

[6] Joe Harris, Algebraic geometry, Graduate Texts in Mathematics, vol. 133, Springer-Verlag, New York, 1992, A first course. MR1182558 (93j:14001)

[7] Robin Hartshorne, Algebraic geometry, Springer-Verlag, New York, 1977, Graduate Texts in Mathematics, no. 52. MR 0463157 (57:3116)

[8] D. Hilbert and A. Hurwitz, Uber die diophantischen Gleichungen vom Geschlecht Null, Acta Math. 14 (1890), no. 1, 217-224. MR.1554798

[9] D. Manocha and J. Canny, Rational curves with polynomial parametrization, Computer Aided Design 23 (1991), no. 9, 653-653.

[10] Tomás Recio and J. Rafael Sendra, Real reparametrizations of real curves, J. Symbolic Comput. 23 (1997), no. 2-3, 241-254, Parametric algebraic curves and applications (Albuquerque, NM, 1995). MR1448697 (98d:14076)

[11] Tomás Recio, J. Rafael Sendra, Luis Felipe Tabera, and Carlos Villarino, Fast computation of the implicit ideal of a hypercircle, Actas de AGGM 2006, 2006, pp. 258-265.

[12] Tomás Recio, J. Rafael Sendra, and Carlos Villarino, From hypercircles to units, ISSAC 2004, ACM, New York, 2004, pp. 258-265. MR.2126952 (2005k:14125)

[13] J. Rafael Sendra and Carlos Villarino, Optimal reparametrization of polynomial algebraic curves, Internat. J. Comput. Geom. Appl. 11 (2001), no. 4, 439-453. MR.1852578 (2002e:14099)

[14] , Algebraically optimal parametrizations of quasi-polynomial algebraic curves, J. Algebra Appl. 1 (2002), no. 1, 51-74. MR 1907738 (2003c:14070)

[15] J. Rafael Sendra and Franz Winkler, Symbolic parametrization of curves, J. Symbolic Comput. 12 (1991), no. 6, 607-631. MR1141543 (93a:14053)

[16] _ Parametrization of algebraic curves over optimal field extensions, J. Symbolic Comput. 23 (1997), no. 2-3, 191-207, Parametric algebraic curves and applications (Albuquerque, NM, 1995). MR1448694 (98d:14077)

[17] Luis Felipe Tabera, Fields of parametrization and optimal affine reparametrization of rational curves, Preprint, arXiv:0810.5595.

[18] Robert J. Walker, Algebraic Curves, Princeton Mathematical Series, vol. 13, Princeton University Press, Princeton, NJ, 1950. MR0033083(11:387e) 
[19] André Weil, Adèles et groupes algébriques, Séminaire Bourbaki, Vol. 5, Soc. Math. France, Paris, 1995, Exp. No. 186, pp. 249-257. MR1603471

[20] http://www.algebra.uni-linz.ac.at/Nearrings/

Departamento de Matemáticas, Estadística y Computación, Universidad de Cantabria, 39071, Santander, Spain

E-mail address: tomas.recio@unican.es

Departamento de Matemáticas, Universidad de Alcalá, 28871, Alcalá de Henares, SPAIN

E-mail address: rafael.sendra@uah.es

Departamento de Matemáticas, Estadística y Computación, Universidad de Cantabria, 39071, SANTANDER, Spain

E-mail address: taberalf@unican.es

Departamento de Matemáticas, Universidad de Alcalá, 28871, Alcalá de Henares, SPAIN

E-mail address: carlos.villarino@uah.es 